\title{
Fisheries-induced disruptive selection
}

\author{
Pietro Landi ${ }^{\mathrm{a}, *}$, Cang Hui ${ }^{\mathrm{b}, \mathrm{c}}$, Ulf Dieckmann ${ }^{\mathrm{d}}$ \\ a Department of Eletronics, Information, and Bioengineering, Politecnico di Milano, Via Ponzio 34/5, 20133 Milano, Italy \\ ${ }^{\mathrm{b}}$ Centre for Invasion Biology, Department of Mathematical Sciences, Stellenbosch University, Matieland 7602, South Africa \\ ${ }^{\mathrm{c}}$ Mathematical and Physical Biosciences, African Institute for Mathematical Sciences, Muizenberg 7945, South Africa \\ ${ }^{\mathrm{d}}$ Evolution and Ecology Program, International Institute for Applied Systems Analysis, Schloßplatz 1, 2361 Laxenburg, Austria
}

\section{H I G H L I G H T S}

- Commonly used fishing policies cause disruptive selection on fish stocks when harvest pressure is high.

- Necessary conditions are adaptive harvest of large individuals and strong life-history tradeoffs of early maturation on growth and fecundity.

- Fisheries-induced disruptive selection is more likely in stocks with a natural predisposition to early maturation.

- Sustainable yield after diversification is far below the MSY attained under lower fishing pressures, for which selection is not disruptive.

\section{A R T I C L E I N F O}

\section{Article history:}

Received 16 June 2014

Received in revised form

7 October 2014

Accepted 14 October 2014

Available online 24 October 2014

Keywords:

Fisheries-induced evolution

Coevolution

Adaptive dynamics

Size at maturation

Evolutionary branching

\begin{abstract}
A B S T R A C T
Commercial harvesting is recognized to induce adaptive responses of life-history traits in fish populations, in particular by shifting the age and size at maturation through directional selection. In addition to such evolution of a target stock, the corresponding fishery itself may adapt, in terms of fishing policy, technological progress, fleet dynamics, and adaptive harvest. The aim of this study is to assess how the interplay between natural and artificial selection, in the simplest setting in which a fishery and a target stock coevolve, can lead to disruptive selection, which in turn may cause trait diversification. To this end, we build an eco-evolutionary model for a size-structured population, in which both the stock's maturation schedule and the fishery's harvest rate are adaptive, while fishing may be subject to a selective policy based on fish size and/or maturity stage. Using numerical bifurcation analysis, we study how the potential for disruptive selection changes with fishing policy, fishing mortality, harvest specialization, life-history tradeoffs associated with early maturation, and other demographic and environmental parameters. We report the following findings. First, fisheries-induced disruptive selection is readily caused by commonly used fishing policies, and occurs even for policies that are not specific for fish size or maturity, provided that the harvest is sufficiently adaptive and large individuals are targeted intensively. Second, disruptive selection is more likely in stocks in which the selective pressure for early maturation is naturally strong, provided life-history tradeoffs are sufficiently consequential. Third, when a fish stock is overexploited, fisheries targeting only large individuals might slightly increase sustainable yield by causing trait diversification (even though the resultant yield always remains lower than the maximum sustainable yield that could be obtained under low fishing mortality, without causing disruptive selection). We discuss the broader implications of our results and highlight how these can be taken into account for designing evolutionarily informed fisheries-management regimes.
\end{abstract}

(c) 2014 Elsevier Ltd. All rights reserved.

\section{Introduction}

The exploitation of renewable resources is a major source of mortality, which can trigger population collapse (Stokes et al., 1993; Hutchings and Reynolds, 2004) and adaptive changes in the life history of harvested species (Palumbi, 2001; Ashley et al., 2003).

\footnotetext{
* Corresponding author. Tel.: + 39022399 3579; fax: + 390223993412.

E-mail addresses: pietro.landi@polimi.it (P. Landi), chui@sun.ac.za (C. Hui), dieckmann@iiasa.ac.at (U. Dieckmann).
}

Indeed, in commercially exploited fish stocks harvest has been recognized a driver of evolutionary adaptations (Law, 2000; Heino and Godø, 2002; Jørgensen et al., 2007; Dieckmann et al., 2009). To date, most studies considering the genetic and phenotypic responses of fish stock to fishing have focused on fisheriesinduced directional selection on life-history traits such as age and size at maturation (Barot et al., 2004; Ernande et al., 2004; de Roos et al., 2006; Gårdmark and Dieckmann, 2006; Dunlop et al., 2009; Poos et al., 2011).

In addition, a fishery itself can adapt, in terms of fishing policy, technological progress, fleet dynamics, and adaptive harvest 
(Salthaug, 2001; Hannesson, 2002; Walters and Martell, 2004). Fishing policies can be selective for both size and maturity stage of individuals in the stock: size selectivity results from mesh-size and gear regulation or from size-specific incentives (Hart and Reynolds, 2002; Fromentin and Powers, 2005), while maturity selectivity may arise when a stock's juveniles and adults are spatially segregated during spawning (Sinclair, 1992; Swain and Wade, 1993; Engelhard and Heino, 2004; Opdal, 2010). Harvest is readily adaptive, because fishers constantly tune their effort and selectivity for maximum profit, targeting stock components that are most profitable to harvest. Such adaptation is relatively fast, leading to a continuously changing selective pressure on the exploited stock. Accordingly, the effect of technological progress on a fishery's sustainability is often assessed while neglecting adaptive responses of the targeted stock (e.g., Dercole et al., 2010).

The coupled dynamics of adaptations in a stock and its fishery can be interpreted as a coevolutionary process, in which one component of the system is biological (the exploited stock) while the other component is economic (the exploiting fishery). In his pioneering work, Heino (1998) approached the stock-fishery system from this coevolutionary perspective: individuals in the considered stock could adapt their age at maturation in response to the selective pressure imposed by harvesting, while fishers adapted their strategy to maximize the sustainable yield on a slower timescale, causing directional selection on the age at maturation.

The interaction between adaptive harvest imposed by a fishery and biological evolution could possibly result in disruptive selection, as suggested by Carlson et al. (2007) and Edeline et al. (2007) and supported by statistical analysis of field data by Edeline et al. (2009). The objective of this study is to provide a first model-based investigation of this phenomenon. For this, we approach the stockfishery system from the coevolutionary perspective, allowing harvest to adapt on the timescale of population dynamics, thus improving on Heino's (1998) timescale-separation assumption, and studying both directional and disruptive selective pressure. Disruptive selection can increase the genetic and/or phenotypic variance of adaptive traits (Gross, 1985; Edeline et al., 2009; Keller et al., 2013), and under some circumstances may even lead to evolutionary branching and dimorphic trait diversification (Maynard Smith, 1966; Geritz et al., 1998). Both impacts may increase a stock's capacity to respond to directional selective pressures (Roff, 1997), and may raise the stock's abundance and yield. Disruptive selection is notoriously difficult to predict and can also have negative effects on the ecosystem in which the fish stock is embedded (Jennings and Kaiser, 1998; Zhou et al., 2010). We conclude our investigation by discussing broad implications of our findings, which might be taken into account for the evolutionarily informed management of fisheries and the design of sustainable fishery policies.

\section{Model and methods}

We use a discretely size-structured life-history model, similar to that employed in Poos et al. (2011) and Bodin et al. (2012), to describe an adaptively harvested fish population divided into three size classes (Fig. 1). Individuals can mature either in the second or in the third size class, and accordingly differ in their sizes at maturation. We refer to the probability of maturing in the second size class as the probability of early maturation, and consider it an adaptive trait constrained by life-history tradeoffs (Roff, 1983; Stearns, 1992). From this stock-fishery model, we derive the stock's basic reproduction ratio in dependence of the adaptive trait, and from this, the evolutionary dynamics of maturation. Using bifurcation analysis (Kuznetsov, 2004) and numerical continuation techniques (Allgower and Georg, 2003), we study the selective pressures exerted on the stock by different levels of fishing mortality and by different levels of selectivity for size and/or maturity. In this way, we assess the potential for fish stocks to experience disruptive selection and thus potentially undergo maturation diversification (Fig. 2).

\subsection{Population dynamics}

We consider a stock in which individuals are classified into three size classes-juveniles, small, and large. An individual can become mature at small size (early maturation) with probability $x$ or at large size with probability $1-x$ (Gross, 1985). The probability of early maturation is analyzed as an adaptive life-history trait under selection. Specifically, we denote by $\mathbf{N}(t)=\left(N_{i}(t)\right)$ the vector of fish abundances at time $t$, with $i=1,2, \tilde{2}, 3$, or $\tilde{3}$ ranging over all stock components (where tilde-subscripts refer to early-maturing individuals). Fig. 1 provides a schematic representation of the considered stock structure.

Newborn juvenile individuals grow into the second size class at rate $r_{1}$. With probability $x$, they are early-maturing, thus growing into stock component $\tilde{2}$, whereas with probability $1-x$ they are late-maturing, thus growing into stock component 2 . Small individuals grow into the third size class at rates $r_{\tilde{2}}$ or $r_{2}$, depending on whether they are early-maturing or late-maturing, respectively. Early-maturing individuals give birth to juveniles in the second and third size classes,

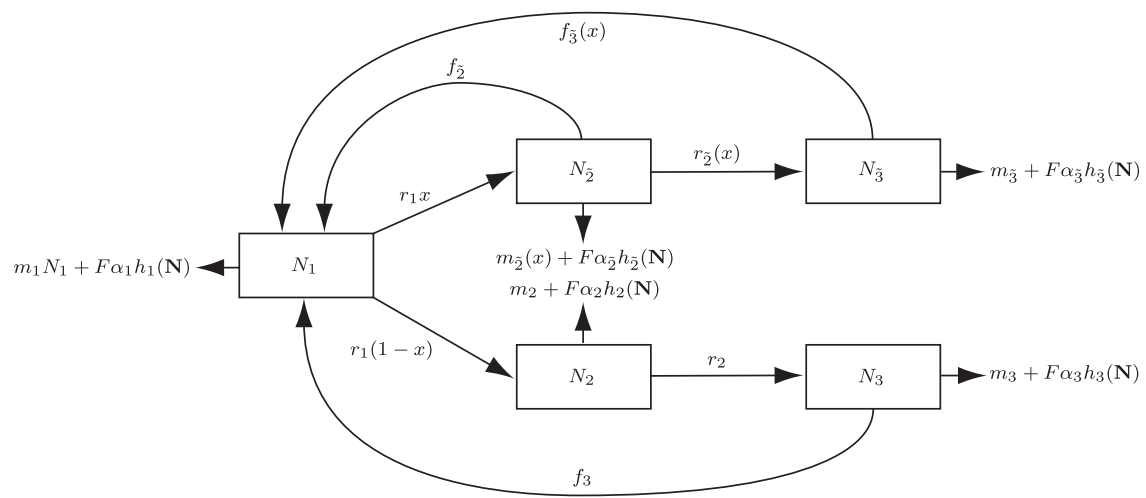

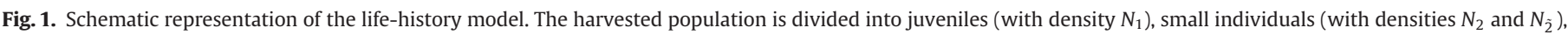

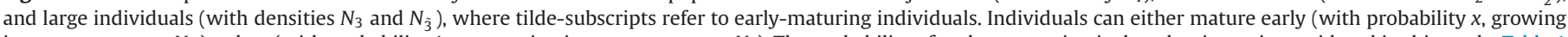

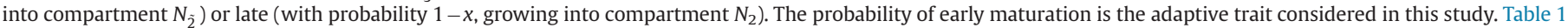
and Section 2 provide further details. 


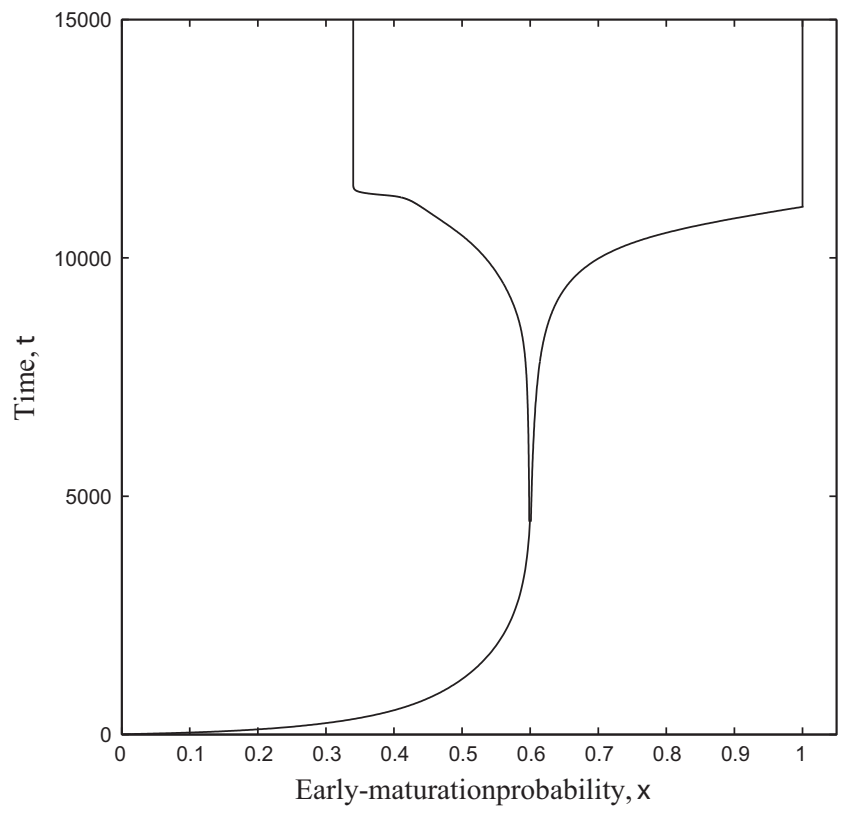

Fig. 2. Model-based illustration of maturation diversification in response to fisheriesinduced disruptive selection. The probability of early maturation, initially set at 0 , gradually converges to a monomorphic evolutionary equilibrium at which selection turns disruptive and evolutionary branching takes place. The resultant two coexisting morphs, which initially are very similar, then diversify, eventually converging to a dimorphic evolutionary equilibrium. Parameters at their reference value (see Table 1) and $F=1.1 \mathrm{yr}^{-1}$.

at rates $f_{\tilde{2}}$ and $f_{\tilde{3}}$, respectively, while late-maturing individuals produce offspring only once they reach the third size class, at rate $f_{3}$. The natural mortality of juveniles is considered to be densitydependent, at rate $m_{1} N_{1}$, indicating resource competition at the juvenile stage, since we assume that juveniles critically depend on scarce resources in the environment. In contrast, we assume that small and large individuals experience density-independent mortality, as the spectrum of their feeding resources is often wide. Specifically, the natural mortality rates are assigned $m_{\tilde{2}}$ and $m_{2}$ in the small size class and $m_{\tilde{3}}$ and $m_{3}$ in the large size class, depending on whether they are early-maturing or late-maturing, respectively.

We assume that early-maturing individuals face several lifehistory tradeoffs, since energy allocation to maturation reduces the energy available for other life-history processes, including growth, survival, and reproduction (Poos et al., 2011; Bodin et al., 2012). We make the simplest possible assumptions for these three tradeoffs, by considering the mortality of small earlymaturing individuals to be increased relative to small latematuring individuals according to $m_{\tilde{2}}(x)=m_{2}\left(1+\beta_{\mathrm{m}} x\right)$, the growth rate of small early-maturing individuals to be decreased relative to small late-maturing individuals according to $r_{\tilde{2}}(x)=r_{2}\left(1-\beta_{\mathrm{r}} x\right)_{+}$, and the fecundity of large early-maturing individuals to be decreased relative to large late-maturing individuals according to $f_{\tilde{3}}(x)=f_{3}\left(1-\beta_{\mathrm{f}} x\right)_{+}$. In each case, the considered costs of early maturation are thus proportional to the probability $x$ of early maturation, with proportionality constants $\beta_{\mathrm{m}}, \beta_{\mathrm{r}}$, and $\beta_{\mathrm{f}}$ measuring the strengths of the respective tradeoffs. The subscript $(\ldots)_{+}$ means that negative values in the parenthesis are mapped to 0 , while positive values remain unchanged. This means that for values of $\beta_{\mathrm{r}}>1$ and $\beta_{\mathrm{f}}>1$ the growth rate $r_{\tilde{2}}(x)$ and the fecundity rate $f_{\tilde{3}}(x)$, respectively, may become zero as $x$ increases, but can never become negative.

Based on these considerations, we obtain the following stockfishery model:

$\dot{N}_{1}=f_{\tilde{2}} N_{\tilde{2}}+f_{\tilde{3}}(x) N_{\tilde{3}}+f_{3} N_{3}-m_{1} N_{1}^{2}-r_{1} N_{1}-F \alpha_{1} h_{1}(\mathbf{N}) N_{1}$,
$\dot{N}_{2}=(1-\chi) r_{1} N_{1}-m_{2} N_{2}-r_{2} N_{2}-F \alpha_{2} h_{2}(\mathbf{N}) N_{2}$,

$\dot{N}_{\tilde{2}}=x r_{1} N_{1}-m_{\tilde{2}}(x) N_{\tilde{2}}-r_{\tilde{2}}(x) N_{\tilde{2}}-F \alpha_{\tilde{2}} h_{\tilde{2}}(\mathbf{N}) N_{\tilde{2}}$,

$\dot{N}_{3}=r_{2} N_{2}-m_{3} N_{3}-F \alpha_{3} h_{3}(\mathbf{N}) N_{3}$,

$\dot{N}_{\tilde{3}}=r_{\tilde{2}}(x) N_{\tilde{2}}-m_{\tilde{3}} N_{\tilde{3}}-F \alpha_{\tilde{3}} h_{\tilde{3}}(\mathbf{N}) N_{\tilde{3}}$,

where $\dot{N}_{i}$ is the time derivative of the abundance $N_{i}$ of each component of the fish stock, while the last term in each equation describes harvest, as explained in the next subsection. All variables and parameters of our stock-fishery model are summarized in Table 1.

\subsection{Fishery dynamics}

Fishing activities imply an extra mortality in each stock component of the form $F \alpha_{i} h_{i}(\mathbf{N}) N_{i}$, where $i$ ranges over all five stock components, $i=1,2, \tilde{2}, 3$, or $\tilde{3}, F$ denotes the fishingmortality rate, the binary vector $\boldsymbol{\alpha}=\left(\alpha_{i}\right)$ characterizes the selective fishing policy according to fish size and maturity, and $h_{i}(\mathbf{N})$ is the relative adaptive harvest of stock component $i$.

We consider ten different fishing policies, with different selectivity according to size and maturity (Ajiad et al., 1999; Law, 2000; Poos et al., 2011; Bodin et al., 2012). These are detailed in Table 2. For example, fishing with no restrictions on size and maturity translates into the vector $\boldsymbol{\alpha}=(1,1,1,1,1)$, while a policy that allows fishing only of mature individuals is represented by the vector $\boldsymbol{\alpha}=(0,0,1,1,1)$. We assume the absolute implementation of the policies: there is perfect selectivity, no by-catch or other non-intended mortality.

The relative adaptive harvest $h_{i}(\mathbf{N})$ of stock component $i$ is described by a power law (Egas et al., 2005),

$h_{i}(\mathbf{N})=\frac{\left(\alpha_{i} w_{i} N_{i}\right)^{\gamma}}{\sum_{j}\left(\alpha_{j} w_{j} N_{j}\right)^{\gamma}}$

with the sum extending over all five stock components $j=1,2, \tilde{2}, 3$, or $\tilde{3}$. In this equation, $w_{i}$ is the weight of a fish in stock component $i$, which is given by the allometric scaling relation $w_{i}=k s_{i}^{\theta}$, where $k$ and $\theta$ are the allometric coefficient and allometric exponent, respectively, and $s_{i}$ is the size of a fish in stock component $i$. Notice that the allometric coefficient cancels in Eq. (1b); its only effect is that of scaling the yield, see (Eqs. (1c) and A.2). The multiplication with fish weights translates the density of individuals into their biomass density. Therefore, the product $w_{i} N_{i}$ is the catch obtainable from harvesting stock component $i$. The parameter $\gamma$ measures the degree of harvest specialization and ranges from 0 to $\infty$. When $\gamma=0$, the harvest is not adaptive and is randomly distributed over all five stock components (in analogy to random foraging). When $\gamma=1$, the relative harvest for each stock component equals the relative catch from that compartment (in analogy to foraging according to the ideal free distribution). When $\gamma$ tends to $\infty$, the harvest is completely focused on the stock component yielding maximum catch (in analogy to optimal foraging). We suggest the value of $\gamma=5$ for a weakly specialized fishery and $\gamma=25$ for a highly specialized fishery (see Table 1 and Fig. 3).

The total sustainable yield of the fishery for a monomorphic stock with trait value $x^{*}$ is given by

$Y_{\mathrm{M}}=\left.\sum_{i} F \alpha_{i} h_{i}\left(\mathbf{N}^{*}\right) N_{i}^{*} w_{i}\right|_{x=x^{*}}$,

that is, the sum of the yields obtained by harvesting the five stock components $i=1,2, \tilde{2}, 3$, and $\tilde{3}$ at the eco-evolutionary equilibrium $\left(\mathbf{N}^{*}, x^{*}\right)$, following the fishing policy $\boldsymbol{\alpha}=\left(\alpha_{i}\right)$. A very similar expression gives the total sustainable yield for a dimorphic stock, see Eq. (A.2) in the Appendix. 
Table 1

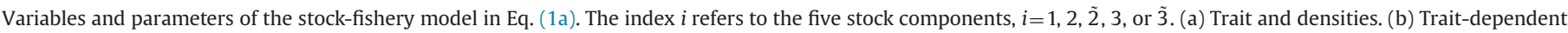
and density-dependent functions. (c) Stock parameters. (d) Fishery parameters.

\begin{tabular}{|c|c|c|c|c|}
\hline & Notation & Description & Reference value & Unit \\
\hline & & Variables & & \\
\hline \multirow[t]{6}{*}{ (a) } & $x$ & Early-maturation probability & NA & NA \\
\hline & $N_{1}$ & Density of juvenile individuals & NA & $\mathrm{km}^{-2}$ \\
\hline & $\mathrm{N}_{2}$ & Density of late-maturing small individuals & NA & $\mathrm{km}^{-2}$ \\
\hline & $N_{\tilde{2}}$ & Density of early-maturing small individuals & NA & $\mathrm{km}^{-2}$ \\
\hline & $N_{3}$ & Density of late-maturing large individuals & NA & $\mathrm{km}^{-2}$ \\
\hline & $N_{\tilde{3}}$ & Density of early-maturing large individuals & NA & $\mathrm{km}^{-2}$ \\
\hline \multirow[t]{5}{*}{ (b) } & $r_{\tilde{2}}(x)$ & Growth rate of early-maturing small individuals & NA & $\mathrm{yr}^{-1}$ \\
\hline & $f_{\tilde{3}}(x)$ & Fecundity rate of early-maturing large individuals & NA & $\mathrm{yr}^{-1}$ \\
\hline & $m_{\tilde{2}}(x)$ & Mortality rate of early-maturing small individuals & NA & $\mathrm{yr}^{-1}$ \\
\hline & $h_{i}(\mathbf{N})$ & Relative adaptive harvest of component $i$ & NA & NA \\
\hline & & Parameters & & \\
\hline \multirow[t]{15}{*}{ (c) } & $r_{1}$ & Growthrate of juvenile individuals & 1 & $\mathrm{yr}^{-1}$ \\
\hline & $r_{2}$ & Growth rate of late-maturing small individuals & 0.8 & $\mathrm{yr}^{-1}$ \\
\hline & $f_{\tilde{2}}$ & Fecundity rate of early-maturing small individuals & 0.8 & $\mathrm{yr}^{-1}$ \\
\hline & $f_{3}$ & Fecundity rate of late-maturing large individuals & 1 & $\mathrm{yr}^{-1}$ \\
\hline & $m_{1}$ & Mortality rate of juvenile individuals & 0.4 & $\mathrm{yr}^{-1}$ \\
\hline & $m_{2}$ & Mortality rate of late-maturing small individuals & 0.3 & $\mathrm{yr}^{-1}$ \\
\hline & $m_{3}$ & Mortality rate of late-maturing large individuals & 0.2 & $\mathrm{yr}^{-1}$ \\
\hline & $m_{\tilde{3}}$ & Mortality rate of early-maturing large individuals & 0.2 & $\mathrm{yr}^{-1}$ \\
\hline & $\beta_{\mathrm{r}}$ & Strength of growth tradeoff & 1 & NA \\
\hline & $\beta_{\mathrm{f}}$ & Strength of fecundity tradeoff & 1 & NA \\
\hline & $\beta_{\mathrm{m}}$ & Strength of mortality tradeoff & 1 & NA \\
\hline & $s_{i}$ & Size of individuals in component $i$ & $0.3 i$ & $\mathrm{~m}$ \\
\hline & $k$ & Allometric coefficient relating size to weight & 0.01 & Tonnes $\mathrm{m}^{-\theta}$ \\
\hline & $\theta$ & Allometric exponent relating size to weight & 3 & NA \\
\hline & $w_{i}$ & Weight of individuals in component $i$ & $k s_{i}^{\theta}$ & Tonnes \\
\hline \multirow[t]{3}{*}{ (d) } & $\alpha=\left(\alpha_{i}\right)$ & Fishing policy & NA & NA \\
\hline & $F$ & Fishing-mortality rate & NA & $\mathrm{yr}^{-1}$ \\
\hline & $\gamma$ & Degree of harvest specialization & 5 & NA \\
\hline
\end{tabular}

Table 2

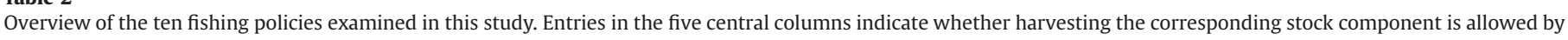

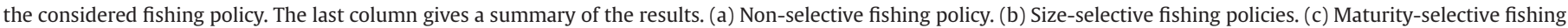
policies. (d) Size- and maturity-selective fishing policies.

\begin{tabular}{|c|c|c|c|c|c|c|c|}
\hline & Fishing policy & Juvenile & Late-maturing small & Early-maturing small & Late-maturing large & Early-maturing large & Results \\
\hline (a) & No regulation & Yes & Yes & Yes & Yes & Yes & Disruptive selection \\
\hline \multirow[t]{5}{*}{ (b) } & Only juvenile & Yes & No & No & No & No & No disruptive selection \\
\hline & Only small & No & Yes & Yes & No & No & No disruptive selection \\
\hline & Only large & No & No & No & Yes & Yes & Disruptive selection $\left(\beta_{\mathrm{r}}<1\right)$ \\
\hline & Juvenile or small & Yes & Yes & Yes & No & No & No disruptive selection \\
\hline & Small or large & No & Yes & Yes & Yes & Yes & Disruptive selection \\
\hline \multirow[t]{2}{*}{ (c) } & Only immature & Yes & Yes & No & No & No & No disruptive selection \\
\hline & Only mature & No & No & Yes & Yes & Yes & Disruptive selection \\
\hline \multirow[t]{2}{*}{ (d) } & Only immature and small & No & Yes & No & No & No & No disruptive selection \\
\hline & Only mature and small & No & No & Yes & No & No & No disruptive selection \\
\hline
\end{tabular}

\subsection{Evolutionary dynamics}

Following Poos et al. (2011) and Bodin et al. (2012), we derive the basic reproduction ratio $R_{0}$, measuring an individual's expected reproductive success in terms of offspring produced during its lifetime. This reproductive success depends both on the trait value of the focal individual and on the other trait values represented in the population. When an individual with trait value $x^{\prime}$ experiences a resident population with trait value $x$ at its demographic equilibrium $\mathbf{N}^{*}(x)$, the focal individual's basic reproduction ratio is given by

$R_{0}\left(x, x^{\prime}\right)=r_{1} D_{1}\left\{\left(1-x^{\prime}\right) r_{2} D_{2} D_{3} f_{3}+x^{\prime}\left[D_{\tilde{2}} f_{\tilde{2}}+r_{\tilde{2}}\left(x^{\prime}\right) D_{\tilde{2}} D_{\tilde{3}} f_{\tilde{3}}\left(x^{\prime}\right)\right]\right\}$, where $D_{1}=\left[m_{1} N_{1}^{*}+r_{1}+F \alpha_{1} h_{1}\left(\mathbf{N}^{*}\right)\right]^{-1}, D_{2}=\left[m_{2}+r_{2}+F \alpha_{2} h_{2}\left(\mathbf{N}^{*}\right)\right]^{-1}$, $D_{\tilde{2}}=\left[m_{\tilde{2}}\left(x^{\prime}\right)+r_{\tilde{2}}\left(x^{\prime}\right)+F \alpha_{\tilde{2}} h_{\tilde{2}}\left(\mathbf{N}^{*}\right)\right]^{-1}, D_{3}=\left[m_{3}+F \alpha_{3} h_{3}\left(\mathbf{N}^{*}\right)\right]^{-1}$, and $D_{\tilde{3}}=\left[m_{\tilde{3}}+F \alpha_{\tilde{3}} h_{\tilde{3}}\left(\mathbf{N}^{*}\right)\right]^{-1}$ are the average durations spent by individuals in each of the five stock components. These are inversely related to the exit rate from those stock components, see Fig. 1 and Eq. (1a). Thus, the product $r_{i} D_{i}$ is the probability that an individual in component $i$ reaches the next size class, while the product $D_{i} f_{i}$ is the expected number of offspring produced by the individual while being in component $i$. The focal individual's basic reproduction ratio $R_{0}\left(x, x^{\prime}\right)$ is a fitness proxy and can be used for evolutionary invasion analysis. Specifically, if $R_{0}\left(x, x^{\prime}\right)>1$, individuals with trait values $x^{\prime}$ can invade and, generically, substitute individuals of a population with resident trait value $x$; otherwise, such invasion is not possible. 
a

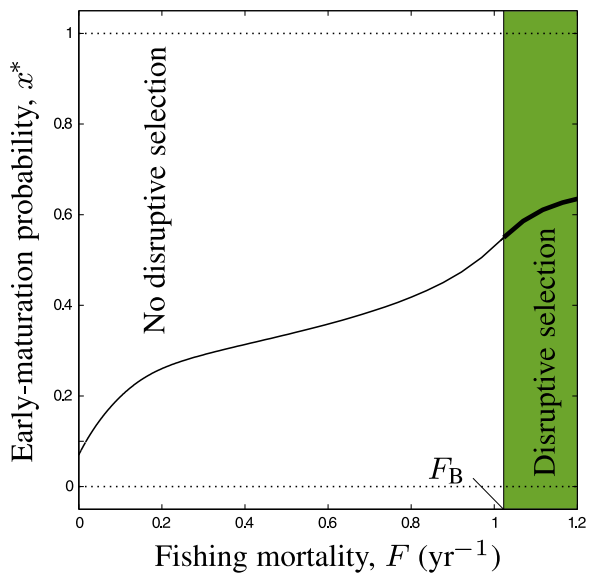

b

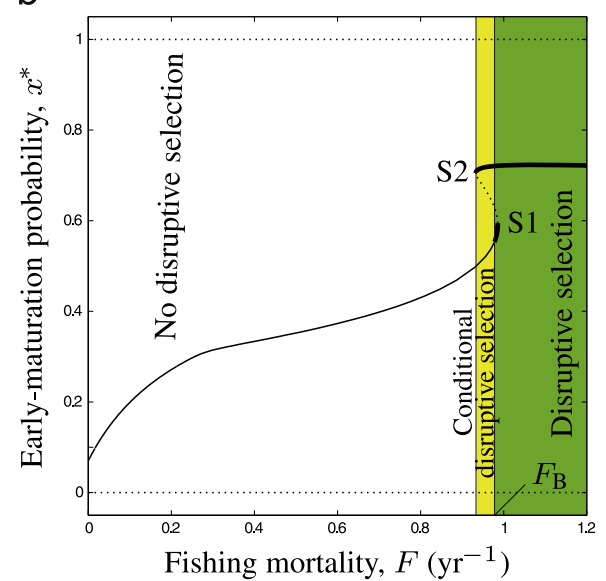

C

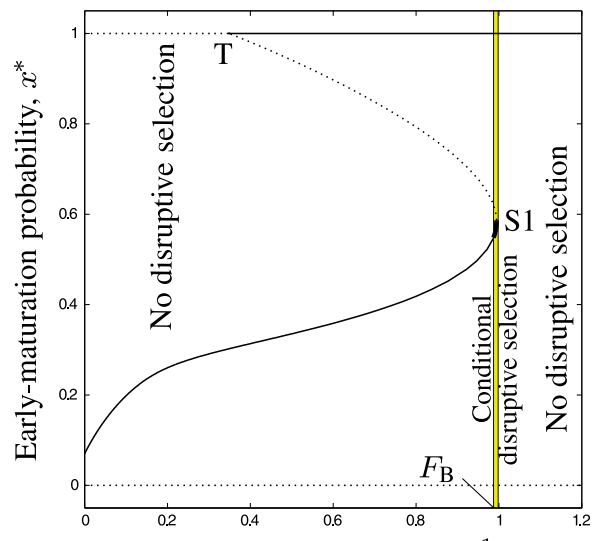

Fishing mortality, $F\left(\mathrm{yr}^{-1}\right)$

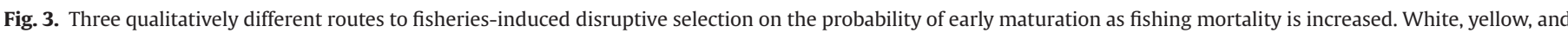

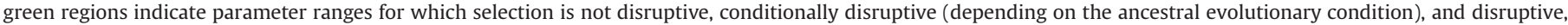

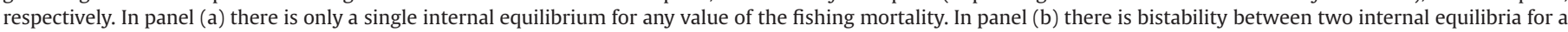

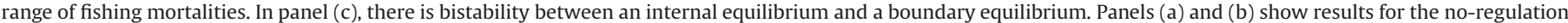

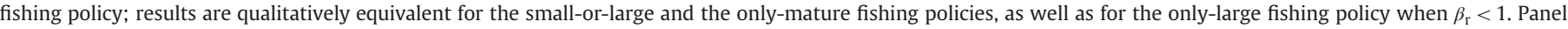

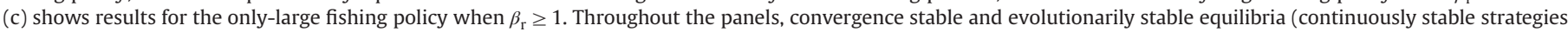

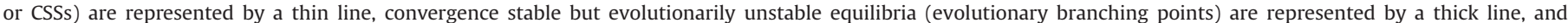

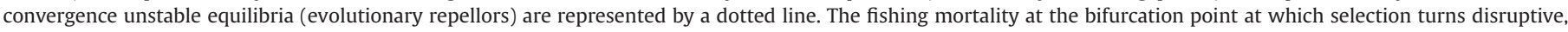

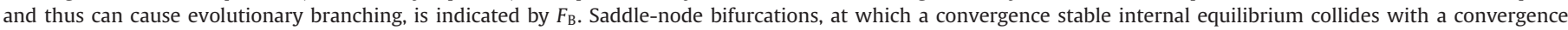

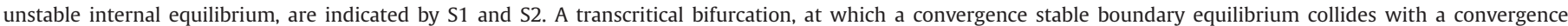

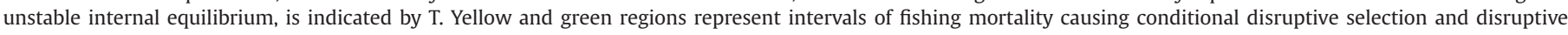

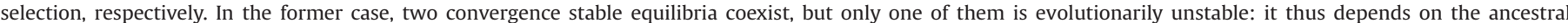

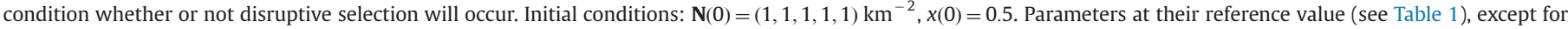
$\gamma=25$ in (b). (For interpretation of the reference to colour in this figure caption, the reader is referred to the web version of this paper.)

The selection gradient

$G(x)=\left.\frac{\partial R_{0}\left(x, x^{\prime}\right)}{\partial x^{\prime}}\right|_{x^{\prime}=x}$

is the slope of the fitness landscape $R_{0}\left(x, x^{\prime}\right)$ around $x$, and measures the strength of the directional selection on $x$. The rate of evolutionary change is proportional to this selection gradient, independent of whether one considers the gradual reshaping of a polymorphic resident trait distribution through selection (as in quantitative genetics theory) or changes in a monomorphic trait distribution through mutation and selection (as in adaptive dynamics theory) (Dieckmann et al., 2006).

Using the selection gradient, we can apply the canonical equation of adaptive dynamics theory (Dieckmann and Law, 1996; Champagnat et al., 2006; Dercole and Rinaldi, 2008), an ordinary differential equation that deterministically approximates the evolutionary dynamics of the adaptive trait $x$. Specifically, the rate of change $\dot{x}$ in the trait value $x$ is proportional to $G(x)$,

$\dot{x} \propto \epsilon G(x)$,

multiplied with half the product of population density, mutation probability, and mutation variance; since the latter three factors are positive, they only regulate the speed of the monomorphic dynamics on the evolutionary timescale, but do not affect the asymptotic evolutionary regime. While the time-scaling parameter $\epsilon$ is used to separate the slow evolutionary timescales from the fast demographic timescale (see also next section). Trait values $0<x^{*}<1$ for which $G\left(x^{*}\right)=0$ are equilibria of the adaptive dynamics, and hence are called evolutionarily singular points. The boundaries $x^{*}=0$ and $x^{*}=1$ are also evolutionary equilibria, even if, generically, the selection gradient $G(x)$ does not vanish at such points (Bodin et al., 2012). Internal equilibria $\left(0<x^{*}<1\right)$ and boundary equilibria $\left(x^{*}=0\right.$ or $x^{*}=1$ ) represent mixed strategies and pure strategies, respectively (see Gross, 1996 for a review).
If the dynamics of the adaptive trait $x$ described by the canonical equation (2c) converges to an evolutionary equilibrium $x^{*}$, that trait value is said to be convergence stable. For internal equilibria, the slope of the fitness landscape then vanishes, and the curvature of the fitness landscape $R_{0}\left(x^{*}, x^{\prime}\right)$ in $x^{\prime}$ determines whether $x^{*}$ is evolutionarily stable or not. If the fitness landscape has a maximum at $x^{*}$ (negative curvature), no mutants can invade and $x^{*}$ is evolutionarily stable: since it is also convergence stable, it is a so-called continuously stable strategy (CSS) (Eshel, 1983; Geritz et al., 1998), characterizing an endpoint of the evolutionary dynamics. Otherwise, if the adaptive dynamics converge to a fitness minimum, it is evolutionarily unstable. Thus, the condition for evolutionary instability is given by

$\left.\frac{\partial^{2} R_{0}\left(x^{*}, x^{\prime}\right)}{\partial x^{\prime 2}}\right|_{x^{\prime}=x^{*}}>0$

If Condition (2d) is satisfied, $x^{*}$ is a fitness minimum, so mutants on both sides of $x^{*}$ can invade. Such mutants and the former residents then coexist on the ecological timescale, forming a new dimorphic resident population. Their traits will experience further disruptive selection and, in the case of asexual populations, are expected to diversify on the evolutionary timescale (Fig. 2). Such diversification can occur also in sexual populations, provided reproductive isolation between the incipient species arises concomitantly (e.g., Keller et al., 2013): here we do not dwell on such complications, which would deserve and require a dedicated separate study, but we assume the concomitant evolution of reproductive isolation. Monomorphic convergence stable singular points satisfying condition (2d) are called evolutionary branching points (Geritz et al., 1997, 1998; Dercole and Rinaldi, 2008). In our analysis below, we will thus test Condition (2d) at monomorphic evolutionary equilibria $x^{*}$ under different fishing policies, as well as for different levels of fishing mortality and different degrees of harvest specialization. 


\subsection{Outline of analysis}

In our further analysis, we use numerical bifurcation analysis and continuation techniques, in an approach similar to that in Landi et al. (2013), to which interested readers are invited to refer for more detailed explanations and discussions.

As the fishing-mortality rate $F$ is the driver of fisheries-induced selection on the stock, we use it as our primary bifurcation parameter. We then extend the analysis by adding a secondary bifurcation parameter, for which we choose $\gamma$, measuring the degree of harvest specialization. In this way, we can assess the effects of fishing, in terms of fishing mortality and fishing specialization, on the occurrence of disruptive selection. To evaluate the generality of results, we also consider as alternative secondary bifurcation parameters the tradeoff strengths $\beta_{\mathrm{r}}, \beta_{\mathrm{f}}$, and $\beta_{\mathrm{m}}$. Eventually, we consider all other demographic and environmental parameters as secondary bifurcation parameters. This procedure will pinpoint the characteristics of stocks that are more likely to experience fisheries-induced disruptive selection, as well as the characteristics of fishing regimes that are more likely to cause such selection. To conclude, we evaluate the effect of fisheries-induced diversification on sustainable yield.

As the analytic form of the demographic equilibrium $\mathbf{N}^{*}(x)$ is unknown for calculating $R_{0}\left(x, x^{\prime}\right)$ in Eq. (2a), we numerically integrate a fast-slow eco-evolutionary dynamics according to Eqs. (1a) and (2c), where the time-scaling parameter $\epsilon=10^{-3}$ regulates the relative speed of the (slow) evolutionary dynamics relative to the speed of the (fast) demographic dynamics (Abrams et al., 1993; Landi et al., 2013). Extensive and systematic numerical analyses of Eq. (1a) reveal that there can only be one nontrivial stable equilibrium $\mathbf{N}^{*}(x)$ for all $0 \leq x \leq 1$. This simplifies the analysis of the adaptive dynamics by ruling out possible bifurcations of the demographic dynamics that could complicate the evolutionary dynamics (Dercole et al., 2002).

We first consider the case without fishing mortality $(F=0)$, with all other parameters set as in Table 1; those parameter values are suitable to model, e.g., the Northern Atlantic Cod stock and are chosen for convenient illustration. Other values have been found to produce qualitatively similar results. We start the fast-slow ecoevolutionary dynamics from the demographic initial condition $\mathbf{N}(0)$ and the evolutionary initial condition $x(0)$ and integrate these dynamics until they converge to the unique eco-evolutionary equilibrium $\left(\mathbf{N}^{*}, x^{*}\right)$. This equilibrium turns out to be a CSS, suggesting that the unharvested stock never experiences disruptive selection and at evolutionary equilibrium has a low probability of early maturation. We then successively consider each of the ten fishing policies listed in Table 2 and examine how the ecoevolutionary equilibrium responds to increasing fishing-mortality rate $F$ (Fig. 3). While doing so, we continuously monitor Condition (2d), which is not satisfied at $F=0$. Depending on the fishing policy, the fishing mortality may reach a threshold $F=F_{\mathrm{B}}$ at which a branching bifurcation occurs, i.e., selection turns disruptive. This means that the initial CSS turns into an evolutionary branching point. We continue to follow this branching bifurcation point while changing both the fishing-mortality rate $F$ and the degree of specialization $\gamma$, obtaining the bifurcation curve in the bivariate $(F, \gamma)$ space that separates regions of disruptive and stabilizing selection (Fig. 4).

\section{Results}

We first examine which fishing policies can cause disruptive selection, then investigate which kind of fish stocks are susceptible to fisheries-induced disruptive selection, and finally, analyze the effects of fisheries-induced diversification on sustainable yield.

\subsection{Which fishing policies can cause fisheries-induced disruptive selection?}

Fig. 3 shows three qualitatively different routes to fisheriesinduced disruptive selection revealed by our model. As fishing mortality is increased in each scenario, the globally convergence stable evolutionarily stable equilibrium at low early-maturation a

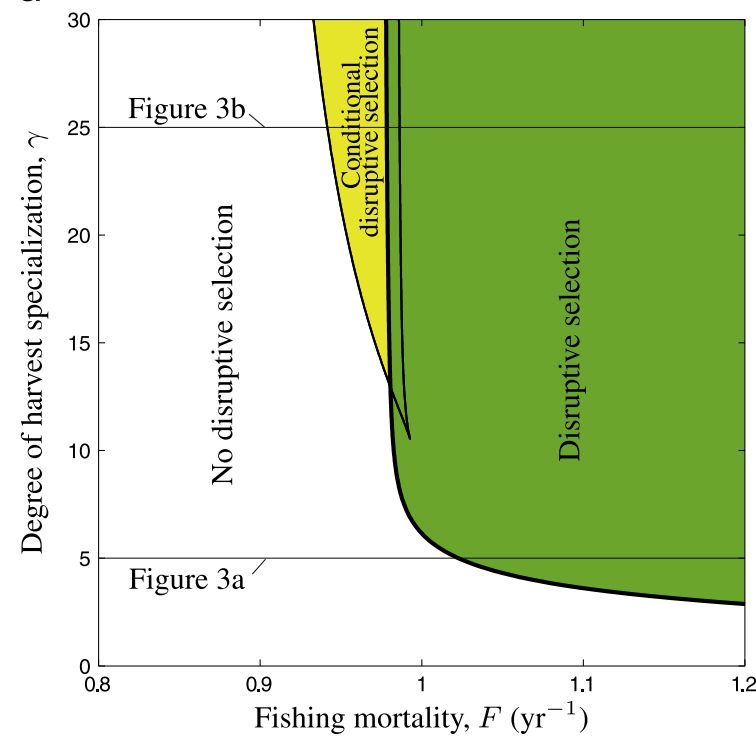

b

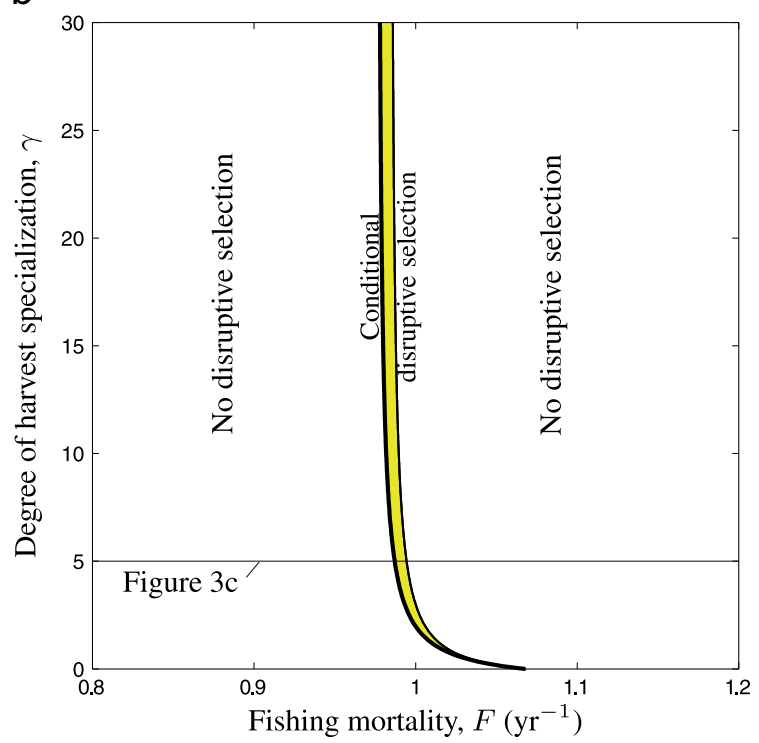

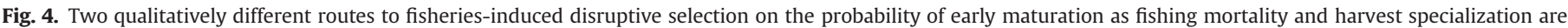

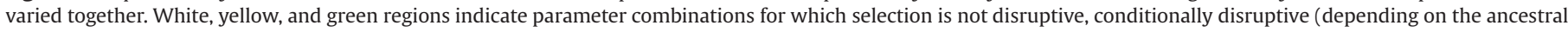

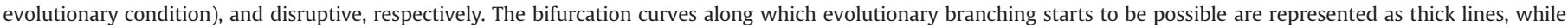

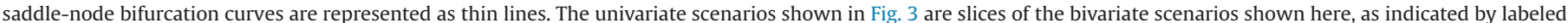

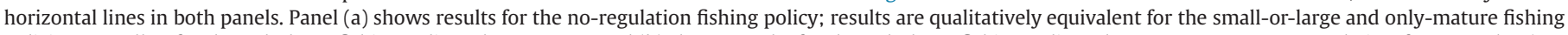

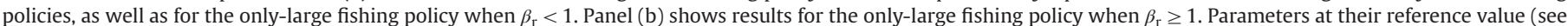
Table 1). (For interpretation of the reference to colour in this figure caption, the reader is referred to the web version of this paper.) 
probability shifts to higher early-maturation probabilities before losing its stability: in scenario (a), it loses its evolutionary stability, while in scenarios (b) and (c), it first loses its global convergence stability and then its evolutionary stability.

Scenario (a): At all levels of fishing mortality, only a single internal equilibrium $\left(0<x^{*}<1\right)$ is present, which is always globally convergence stable. Both boundary equilibria $\left(x^{*}=0\right.$ and $x^{*}=1$ ) are convergence unstable. The early-maturation probability increases with fishing mortality. At high levels of fishing mortality $\left(F>F_{\mathrm{B}}\right.$; green region), the internal equilibrium loses its evolutionary stability, so selection becomes disruptive. This scenario occurs for four of the ten studied fishing policies: it applies to the no-regulation, small-or-large, and only-mature fishing policies, as well as to the only-large fishing policy when $\beta_{\mathrm{r}}<1$ (see below).

Scenario (b): At intermediate levels of fishing mortality $\left(F_{\mathrm{S} 1}<F<F_{\mathrm{S} 2}\right)$, two alternative convergence stable internal equilibria are present. At either end of the interval, two different saddlenode bifurcations occur $\left(F=F_{\mathrm{S} 1}\right.$ and $F=F_{\mathrm{S} 2}$, with $\left.F_{\mathrm{S} 2}<F_{\mathrm{S} 1}\right)$, annihilating one of the convergence stable internal equilibria. The upper internal convergence stable equilibrium is always an evolutionary branching point, whereas the lower internal convergence stable equilibrium is an evolutionary branching point only for $F>F_{\mathrm{B}}$. In this scenario, selection is conditionally disruptive, depending on the ancestral condition $x(0)$, when $F_{\mathrm{S} 2}<F<F_{\mathrm{B}}$ (yellow region), as the early-maturation probability can either converge to the upper internal convergence stable equilibrium (which is an evolutionary branching point; thick line) or to the lower internal convergence stable equilibrium (which is a CSS; thin line). Selection is always disruptive for $F>F_{\mathrm{B}}$ (green region), no matter which one of the two internal convergence stable equilibria is reached from the ancestral condition. This scenario occurs for four of the ten studied fishing policies: it applies to the no-regulation, small-or-large, and only-mature fishing policies, as well as to the only-large fishing policy when $\beta_{\mathrm{r}}<1$ (see below). Notice that this set of fishing policies is the same as for scenario (a), highlighting that it depends on model parameters other than fishing mortality which of the two scenarios applies.

Scenario (c): At intermediate levels of fishing mortality $\left(F_{\mathrm{T}}<F<F_{\mathrm{S} 1}\right)$, a convergence stable internal equilibrium coexists with a convergence stable boundary equilibrium. At either end of the interval, two different bifurcations occur, annihilating one of the convergence stable equilibria. First, a transcritical bifurcation happens at $F=F_{\mathrm{T}}$, when the convergence unstable internal equilibrium (dotted line) collides with the convergence stable boundary equilibrium $x^{*}=1$ (thin line), exchanging their convergence stability. Second, a saddle-node bifurcation happens at $F=F_{\mathrm{S} 1}$ when the same convergence unstable internal equilibrium (dotted line) collides with the internal evolutionary branching point (thick line). In this scenario, selection is conditionally disruptive, depending on the ancestral condition, when $F_{\mathrm{B}}<F<F_{\mathrm{S} 1}$ (yellow region): if the ancestral condition $x(0)$ lies below the convergence unstable internal equilibrium (dotted line), the early-maturation probability converges to the convergence stable internal equilibrium (which is an evolutionary branching point; thick line), so selection becomes disruptive. In contrast, if the ancestral condition lies above the convergence unstable internal equilibrium, the early-maturation probability converges to the boundary equilibrium $x^{*}=1$, where selection cannot be disruptive, as trait values $x>1$ are unfeasible. This scenario occurs for only one fishing policy: it applies to the only-large fishing policy when $\beta_{\mathrm{r}} \geq 1$ (see below).

These results imply that fisheries-induced disruptive selection is readily caused by commonly used fishing policies, namely those targeting large adult and mature individuals while protecting juveniles and immature individuals (Fenberg and Roy, 2008; Darimont et al., 2009). By contrast, scenarios (a)-(c) cannot occur for six of the ten studied fishing policies: this applies to the only-juvenile, only-small, juvenile-or-small, only-immature, only-immature-and-small, and only-mature-and-small fishing policies. Consequently, these six types of fisheries can never cause fisheries-induced disruptive selection (see Table 2).

We can now expand our analysis by considering the effect of harvest specialization on disruptive selection. For this, we need to continue the aforementioned bifurcations in the bivariate $(F, \gamma)$ space, obtaining the bivariate disruptive-selection scenarios shown in Fig. 4. These plots provide a full qualitative characterization of the effects of fishing-in terms of policy, fishing mortality, and the degree of harvest specialization-on disruptive selection. Notice that the univariate scenarios shown in Fig. 3 can be understood as slices, for fixed degrees of harvest specialization $\gamma$, of the bivariate scenarios shown in Fig. 4. In particular, Fig. 3a and b are slices of Fig. 4a for two different degrees of harvest specialization, while Fig. $3 \mathrm{c}$ is a slice of Fig. $4 \mathrm{~b}$. For this reason, we only have two bivariate scenarios, one applying to the noregulation, small-or-large, and only-mature fishing policies, as well as to the only-large fishing policy when $\beta_{\mathrm{r}}<1$ (Fig. 4a) and the other one applying to the only-large fishing policy when $\beta_{\mathrm{r}} \geq 1$ (Fig. 4b).

From these bivariate scenarios we obtain the following results. First, disruptive selection occurs only for high levels of fishing mortality. Second, harvest specialization promotes disruptive selection: at high values of $\gamma$, selection turns disruptive already for lower fishing mortalities (this effect becomes saturated as harvest specialization is increased). Third, random, and thus nonadaptive, harvest $(\gamma=0)$ prohibits disruptive selection, demonstrating that adaptive harvest is a necessary condition for the occurrence of fisheries-induced disruptive selection. Fourth, all four fishing policies causing disruptive selection target large individuals, which therefore is a second necessary condition for the occurrence of fisheries-induced disruptive selection.

\subsection{Which kinds of fish stocks are susceptible to fisheries-induced disruptive selection?}

To find out which kinds of stocks are susceptible to fisheriesinduced disruptive selection, we carry out a sensitivity analysis for the two fisheries-induced disruptive selection scenarios in Fig. 4 with respect to the tradeoff strengths $\beta_{\mathrm{r}}, \beta_{\mathrm{f}}$, and $\beta_{\mathrm{m}}$ (Figs. 5 and 6 ), continuing all detected bifurcations in the $\left(F, \beta_{j}\right)$ spaces, with $j$ spanning all three tradeoffs, $j=\mathrm{r}$, $\mathrm{f}$, or $\mathrm{m}$.

We find that the univariate and bivariate scenarios for disruptive selection under the only-large fishing policy (Figs. 3c and $4 \mathrm{~b}$, respectively) occur only when $\beta_{\mathrm{r}} \geq 1$ (Fig. 5), that is, when the growth tradeoff is very strong. Fig. $3 c$ shows that for $\beta_{\mathrm{r}}=1$ and large fishing mortality $F$ only the boundary equilibrium $x^{*}=1$ exists: at that evolutionary equilibrium, $r_{\tilde{2}}=0$, i.e., early-maturing individuals stop growing. The stock will then be composed of only juveniles and early-maturing small individuals, so that, under the considered only-large fishing policy, it escapes all fishing. Such a complete escape from fishing seems clearly unrealistic: at the very least, it would trigger a switch to a different fishing policy. Fig. 5 shows that when $\beta_{\mathrm{r}} \geq 1$, this unrealistic situation occurs for even smaller fishing mortalities $F$. We therefore discard the scenarios in Figs. $3 \mathrm{c}$ and $4 \mathrm{~b}$ as unrealistic for larger fishing mortalities $F$. In addition, these scenarios can never cause unconditional fisheries-induced disruptive selection, while the conditions under which they cause conditional fisheries-induced disruptive selection are very restrictive, as the narrowness of the yellow regions in Figs. 3c, 4b, and 5 documents. For these reasons, we focus our further analyses on the scenarios in Figs. 3a and b, and 4a, which also cover the only-large fishing policy for $\beta_{\mathrm{r}}<1$. For the purpose of illustration, we consider the no-regulation fishing policy, as all effects shown in Fig. 6 are qualitatively equivalent for all four 


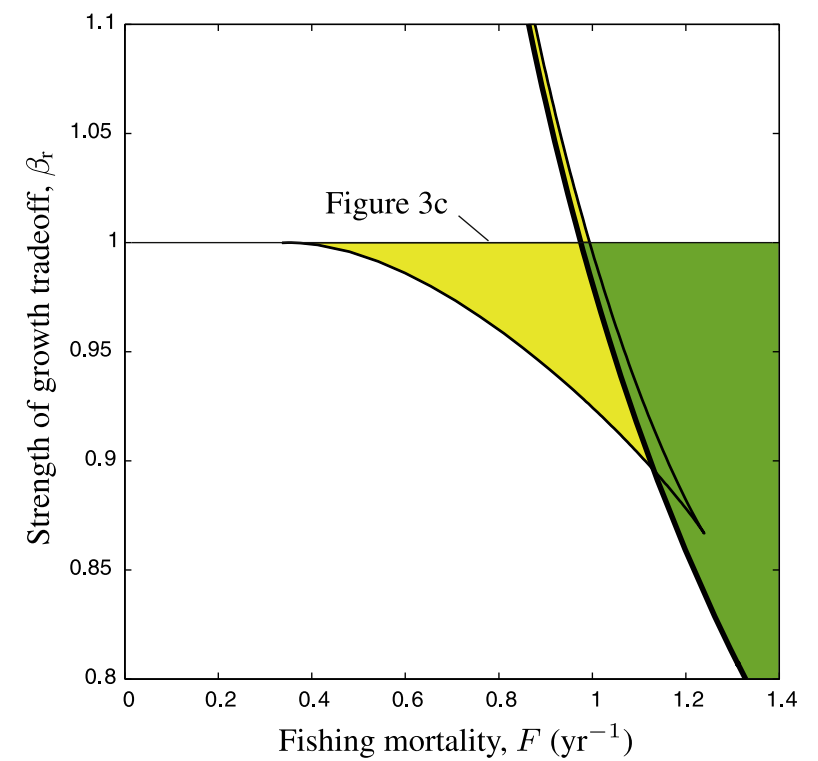

Fig. 5. Limited realism and generality of the fisheries-induced disruptive selection scenario for the only-large fishing policy with $\beta_{\mathrm{r}} \geq 1$. As explained in the text, this scenario unrealistically allows the stock to escape all fishing by maturing early. Also, it can never cause unconditional fisheries-induced disruptive selection, and can cause conditional fisheries-induced disruptive selection only for the restrictive conditions in the narrow yellow band in the upper part of the figure. Hence, the more realistic and general scenario is that in Fig. 4a. Colors and lines as in Fig. 4. Parameters at their reference value (see Table 1). (For interpretation of the reference to colour in this figure caption, the reader is referred to the web version of this paper.)

fishing policies that can cause disruptive selection in the scenarios in Figs. 3a and b, and 4a (no-regulation, small-or-large, onlymature fishing policies, as well as only-large fishing policy when $\left.\beta_{\mathrm{r}}<1\right)$.

Relaxing the tradeoffs in growth and fecundity restrains disruptive selection (Fig. $6 \mathrm{a}$ and b). Disruptive selection is impossible when either one of these tradeoffs is absent (i.e., when $\beta_{\mathrm{r}}=0$ or $\beta_{\mathrm{f}}=0$; Fig. $6 \mathrm{a}$ and $\mathrm{b}$ ): this means that the joint presence of growth and fecundity tradeoffs of early maturation is a necessary condition for the occurrence of disruptive selection. In contrast, relaxing the tradeoff in mortality promotes disruptive selection (Fig. 6c), and disruptive selection is still possible even when this tradeoff is absent (i.e., when $\beta_{\mathrm{m}}=0$; Fig. 6c).

To identify other characteristics of fish stocks that are susceptible to fisheries-induced disruptive selection, we now analyze the effects of all demographic and environmental parameters. In this way, we obtain the following findings. First, the juvenile growth rate $r_{1}$ and the juvenile mortality rate $m_{1}$ do not have any effect on disruptive selection (not illustrated). This is because all individuals have to pass through the juvenile stage in a way that cannot be affected by their adaptive trait. Second, disruptive selection is promoted by increasing the mortality rate $m_{3}$ of large individuals (Fig. 6d), the allometric exponent $\theta$ relating size to weight (Fig. 6e), and the fecundity rate $f_{\tilde{2}}$ of early-maturing small individuals (Fig. 6f). Increasing the first two parameters can reduce the time individuals spend in the large size class, lowering that class' contribution to fitness according to Eq. (2a). Equivalently, increasing the last parameter increases the contribution of small individuals to fitness. Hence, all three cases select for earlier maturation: this, in turn, strengthens the impacts of the considered tradeoffs and thereby promotes disruptive selection. Third, by contrast, disruptive selection is restrained by increasing the mortality rate $m_{2}$ of late-maturing individuals (Fig. 6g), the growth rate $r_{2}$ of latematuring small individuals (Fig. $6 \mathrm{~h}$ ), and the fecundity rate $f_{3}$ of late-maturing large individuals (Fig. 6i). Hence, all three cases select for later maturation; this, in turn, weakens the impacts of the considered tradeoffs and thereby restrains disruptive selection.

In general, therefore, selection is more likely to be disruptive if large individuals make a smaller contribution to fitness according to Eq. (2a), that is, when selection for early maturation is naturally strong. Then the resultant high early-maturation probability will strengthen the impact of life-history tradeoffs in growth and fecundity so as to promote fisheries-induced disruptive selection.

\subsection{What are the effects of diversification on sustainable yield?}

We now analyze the situation in which, after diversification, two coexisting resident populations exhibit alternative trait values $x$ and $y$ close to the evolutionary equilibrium $x^{*}$ of the monomorphic stock. These two coexisting resident traits then diverge on the evolutionary timescale, under the continuous influence of disruptive selection, and eventually settle onto a dimorphic evolutionary equilibrium $\left(x_{\mathrm{D}}^{*}, y_{\mathrm{D}}^{*}\right)$ (Fig. 2). The corresponding dimorphic evolutionary dynamics are specified in the Appendix. In principle, a dimorphic evolutionary equilibrium might be an evolutionary branching point for one or both of the diverged populations. However, in our case, $y_{D}^{*}$ always equals 1 , i.e., individuals of one resident population are always maturing as early as possible; as highlighted above, such a boundary equilibrium cannot be an evolutionary branching point. By contrast, $x_{\mathrm{D}}^{*}$ is evolutionarily stable. Therefore, no further diversification is possible at the dimorphic evolutionary equilibrium.

Once the dimorphic evolutionary equilibrium is attained, the stock's density, and thus its sustainable yield, changes relative to the monomorphic evolutionary equilibrium. Using Eqs. (1c) and (A2), we can evaluate the sustainable yield for different fishingmortality rates $F$ (Fig. 7), again using numerical continuation. We thereby find that, for $0<F<F_{\mathrm{B}}$ (where $F_{\mathrm{B}}$ again denotes the fishing mortality rate at the branching bifurcation) the stock stays at its monomorphic evolutionary equilibrium $x^{*}$, while for $F>F_{\mathrm{B}}$ the monomorphic evolutionary equilibrium becomes evolutionarily unstable, and the stock, following a two-dimensional canonical equation, Eq. (A.1), converges to $\left(x_{\mathrm{D}}^{*}, y_{\mathrm{D}}^{*}\right)$. Note that discontinuities in yield at $F=F_{\mathrm{B}}$ shown in Fig. 7a and b are not surprising, as the outcome of the evolutionary dynamics does not vary continuously with the fishing mortality $F$ across the branching bifurcation.

After diversification, the sustainable yield can slightly increase, but only for the only-large fishing policy when $\beta_{\mathrm{r}}<1$. Even then, it remains far below the maximum sustainable yield, defined by the peaks in Fig. 7a and b. When the fishing-mortality rate $F$ is increased beyond $F_{\mathrm{B}}$, the sustainable yield continuously declines toward zero for the no-regulation, small-or-large and only-mature fishing policies, but remains practically constant (after slightly increasing) for the only-large fishing policy when $\beta_{\mathrm{r}}<1$. This is because the only-large fishing policy, in contrast to the other three fishing policies, does not allow fishing on the early-maturing small individuals in stock component $\tilde{2}$, which are vital for sustaining the stock under very high exploitation rates.

\section{Discussion}

Human exploitation of fish stocks as renewable resources often causes massive mortality. This alters the fitness landscapes of the exploited fish stocks, which in turn may cause adaptive responses of the stocks' phenotypic and genotypic variability (Hutchings and Fraser, 2008). In general, coexisting life-history strategies and corresponding polymorphism can be induced and maintained by negatively frequency-dependent selection (as, for example, in the size at maturation of male coho salmon; Gross, 1985). In this study, 
a

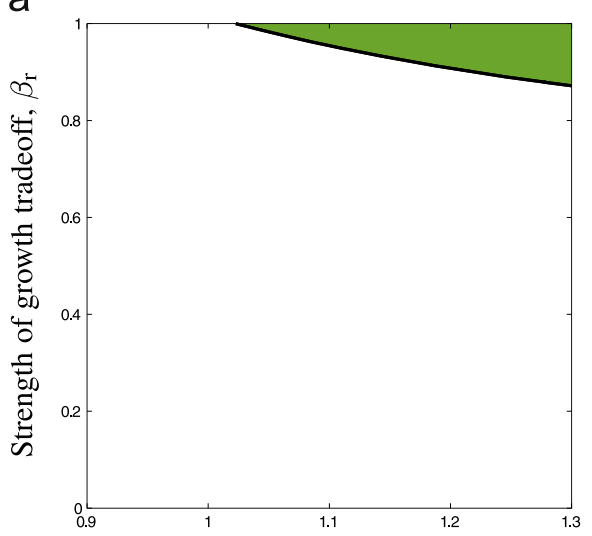

Fishing mortality, $F\left(\mathrm{yr}^{-1}\right)$

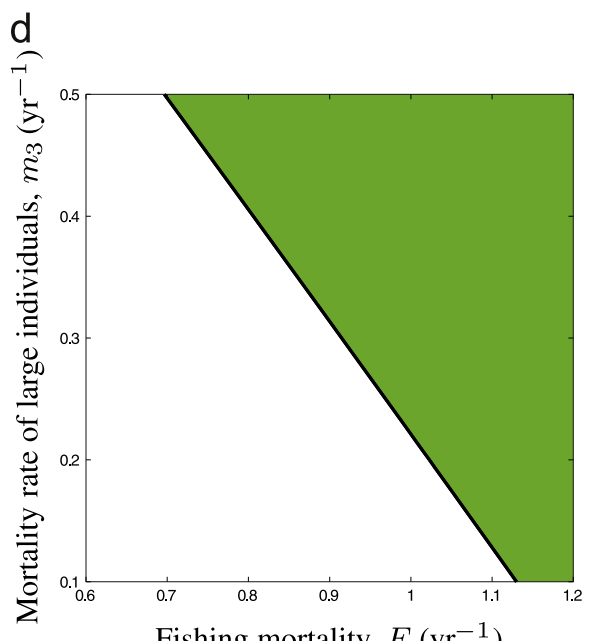

Fishing mortality, $F\left(\mathrm{yr}^{-1}\right)$

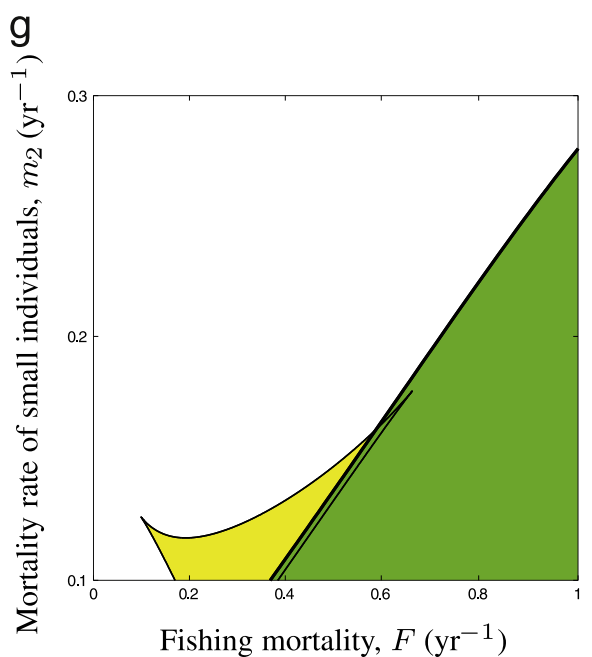

b

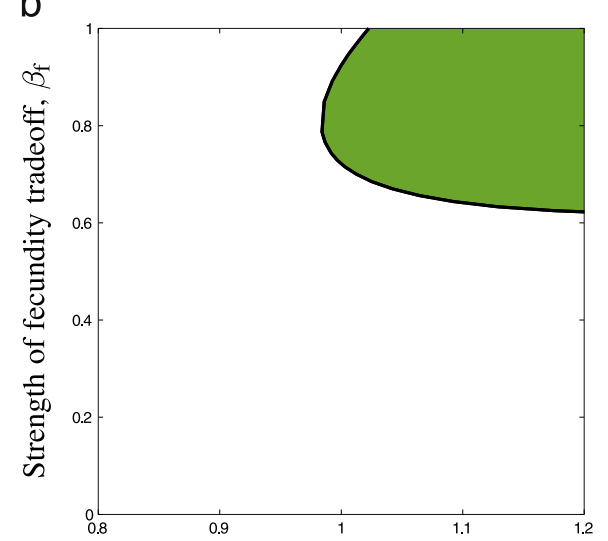

Fishing mortality, $F\left(\mathrm{yr}^{-1}\right)$

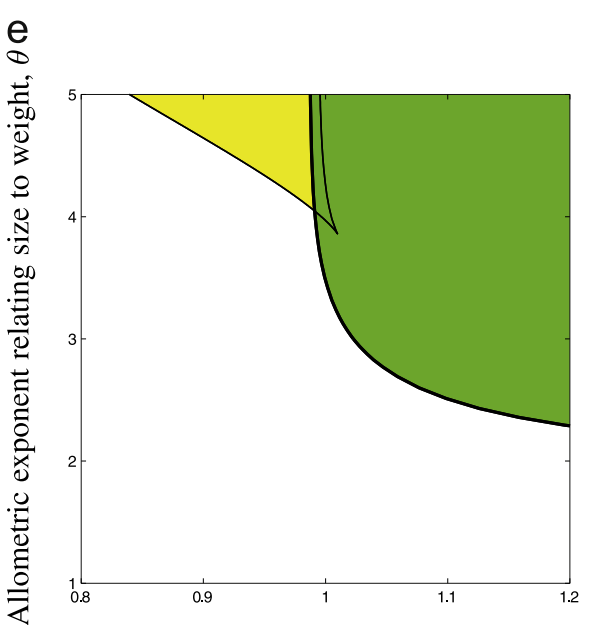

Fishing mortality, $F\left(\mathrm{yr}^{-1}\right)$

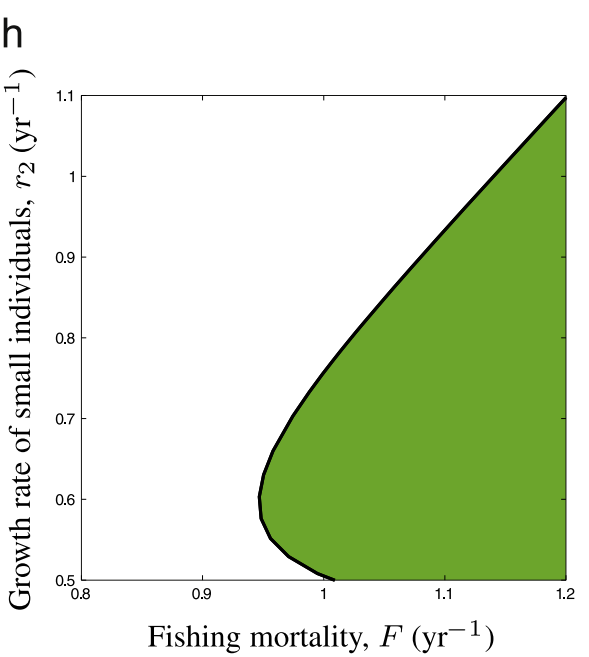

C

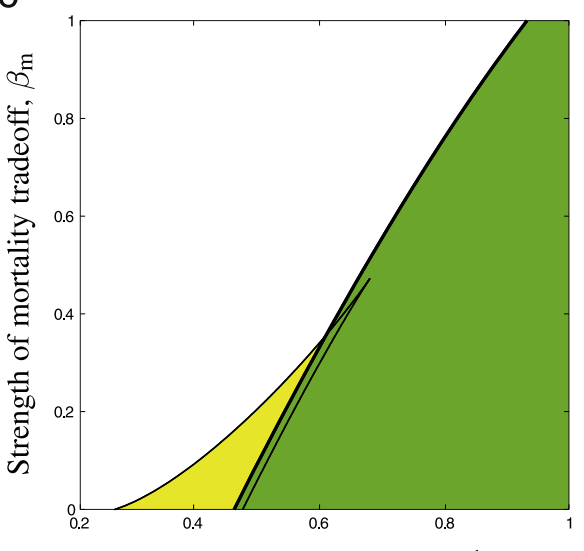

Fishing mortality, $F\left(\mathrm{yr}^{-1}\right)$

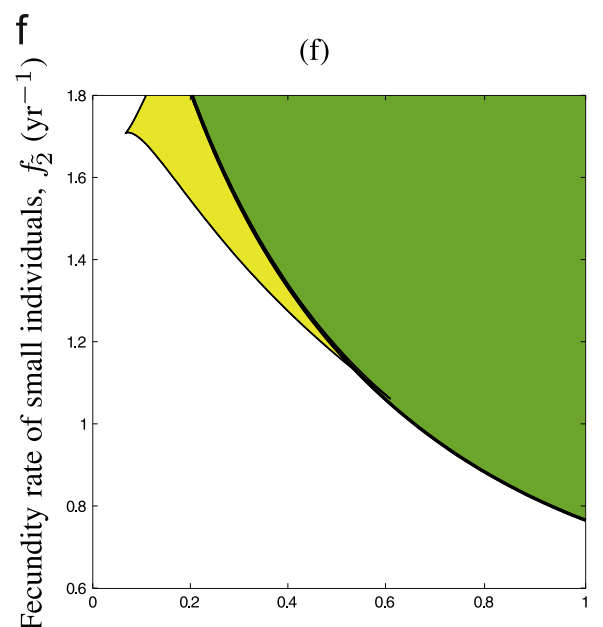

Fishing mortality, $F\left(\mathrm{yr}^{-1}\right)$

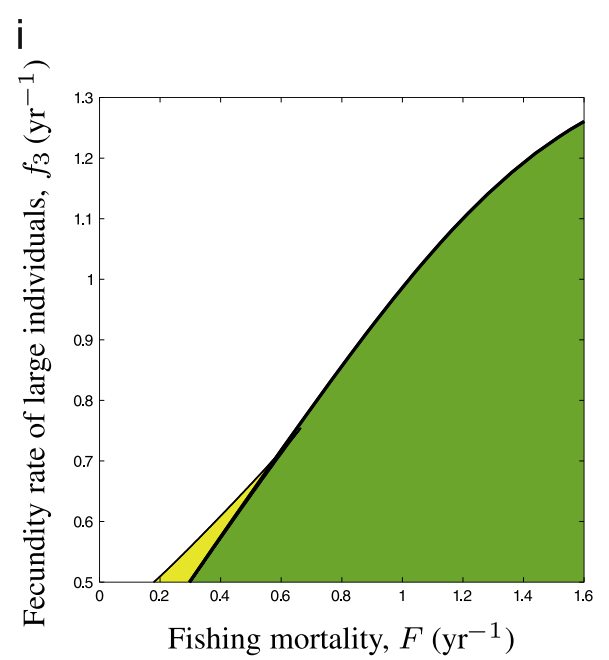

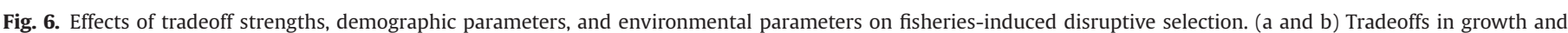

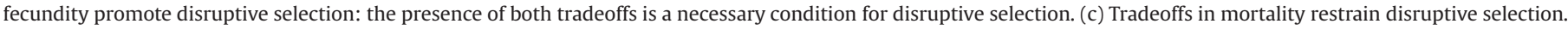

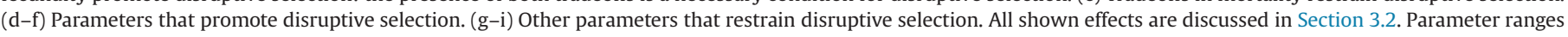

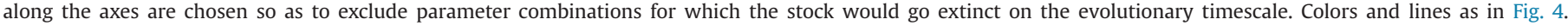
Parameters at their reference value (see Table 1).

we have considered a life-history trait given by a discrete probabilistic reaction norm for the size at maturation (Dieckmann and Heino, 2007), representing the amount of energy allocated to early maturation. In particular, we assume limited energy availability only in the juvenile stage (which translates into densitydependent mortality due to resource competition), while no such limitations are present for the small and large life stages. However, early maturation imposes limitations for other physiological activities, such as growth, reproduction, and survival. For this reason, we introduced trait-dependent tradeoffs: the more energy is allocated to early maturation, the higher the resultant costs in terms of reduced growth, survival, and reproduction. Here we have 
a

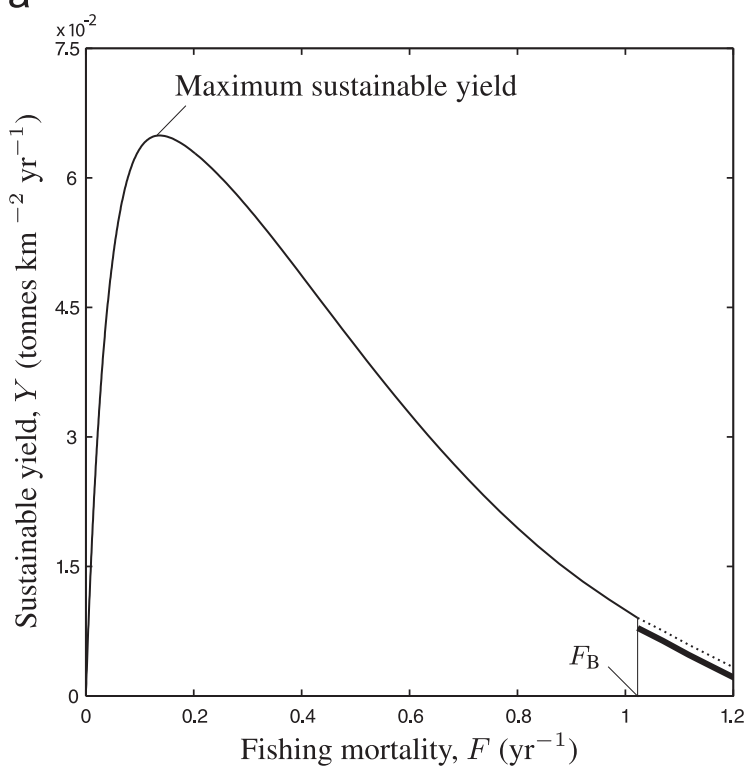

b

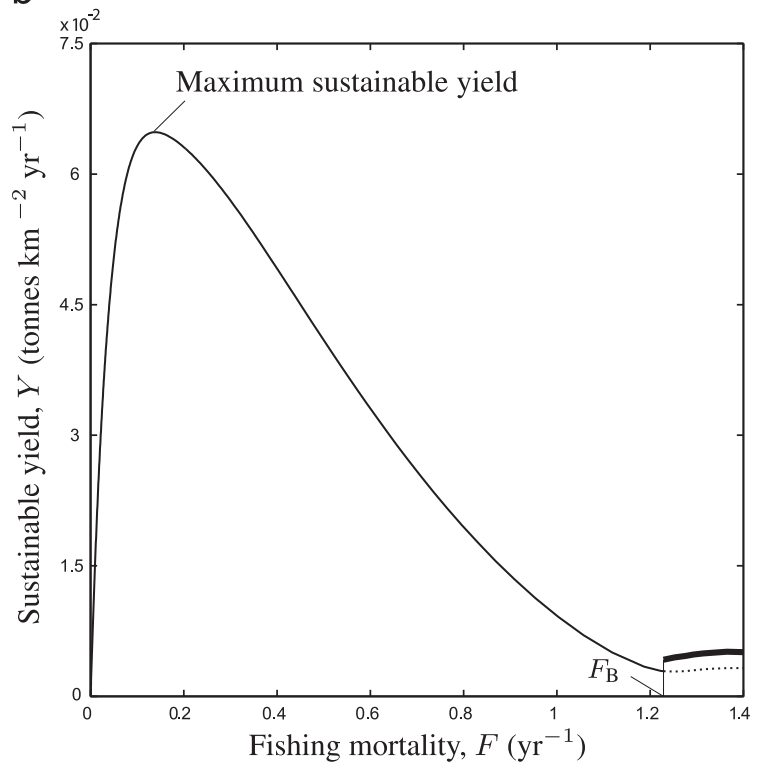

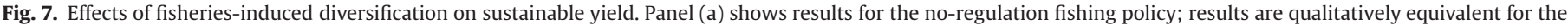

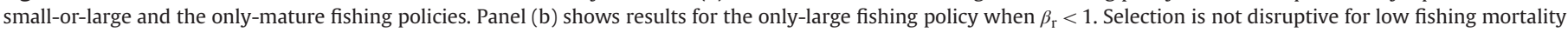

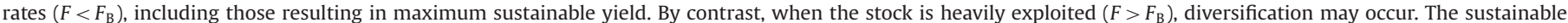

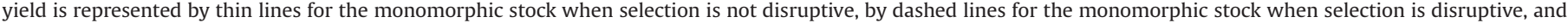

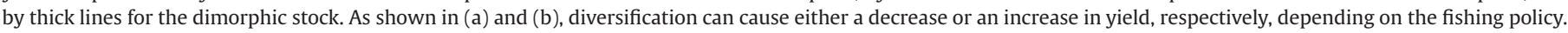
Parameters at their reference value (see Table 1), except for $\beta_{\mathrm{r}}=0.85$ in (b).

demonstrated that fisheries-induced selection on such a trait can be disruptive: this means not only that dimorphism in fish populations can be maintained, but also that such dimorphism may evolve de novo (Keller et al., 2013), thereby giving rise to a coexistence of maturation strategies (Gross, 1996). Several empirical studies have argued the possibility of disruptive selection in fish populations through the interplay of natural selection and adaptive harvesting (Carlson et al., 2007; Edeline et al., 2007, 2009): here we have systematically analyzed, for the first time, under which specific conditions such disruptive selection may arise.

Fishing imposes a strong selective pressure for early maturation, even though this is accompanied by increased physiological costs via life-history tradeoffs. In our model, such selection forces first give rise to a convergence stable mixed strategy, consistent with the argument by Carlson et al. (2007) that natural selection and fisheries-induced selection often act in opposite directions and hence produce strongly stabilizing selection. We have found that, however, with sufficiently strong tradeoffs in growth and fecundity, this convergence stable mixed strategy can become evolutionarily unstable, implying disruptive selection and enabling the coexistence of two maturation strategies, consistent with the argument by Edeline et al. (2009) that fisheries-induced disruptive selection tends to increase trait variance. Specifically, a harvested stock may split into two life-history types: one exploits the advantages of early maturation, while the other reduces the losses imposed by growth and fecundity tradeoffs. By contrast, an analogous life-history tradeoff in mortality has the opposite effect: disruptive selection is enhanced when this tradeoff is relaxed. Moreover, we have shown that strong growth and fecundity tradeoffs both act as indispensable prerequisites for disruptive selection (Fig. 6a and b), while a weak mortality tradeoff merely serves as a dispensable promotor of disruptive selection (Fig. 6c).

In addition to strong life-history tradeoffs in both growth and fecundity, we have identified two other necessary conditions for a stock-fishery system to experience disruptive selection: (i) fishing policies that target large individuals, and (ii) adaptive harvesting that adjusts the harvest distribution for optimal benefit (Fig. 4). Ultimately, these two conditions emerge from the same mechanism described in the previous paragraph. For selection to turn disruptive, the impact of growth and fecundity tradeoffs must become large, and this happens more readily when the probability of early maturation becomes high. Harvesting a stock's large individuals, as happens through many widely adopted fishing policies (Table 2), increases the directional selection pressure toward early maturation, as recurrently highlighted by earlier studies (e.g., Law, 1979; Law and Grey, 1989; Abrams and Rowe, 1996). Moreover, when harvesting is adaptive, a fishery behaves similar to an optimally foraging predator that maximizes its intake rate (e.g., Egas et al., 2005): this tends to increase the mortality of large individuals, as these are more profitable to harvest (Fenberg and Roy, 2008; Darimont et al., 2009). Due to economic, technological, or regulation reasons, large and mature individuals are often the target of fishing. For example, the trawl fisheries of North Sea sole and plaice mainly target only large individuals; a scientific gill net fishery in Windermere, UK, has targeted large individuals of Northern pike for four decades (Carlson et al., 2007; Edeline et al., 2009). Size-selective gill nets were also used for catching striped bass in Maryland during 1950s (Mansueti, 1961); sizeselective harvesting of British Columbia pink salmon has been recorded since 1950 (McAllister and Peterman, 1992). Mature individuals of Norwegian spring-spawning herring have been harvested at their spawning grounds throughout the 20th century, while mature individuals of Northeast Arctic cod have been harvested during their spawning migration until the mid-20th century (Poos et al., 2011). Therefore, adaptive harvesting under policies that allow the targeting of large individuals alters natural adaptive landscapes in a way that selects for increased reproductive investment early in life. This, in turn, reduces somatic growth and fecundity later in life through life-history tradeoffs (Edeline et al., 2007), and thereby strengthens the mechanism that leads to disruptive selection. Poos et al. (2011) and Bodin et al. (2012) have considered a rather similar model, yet without considering adaptive harvesting and trait-dependent tradeoffs: this explains why 
disruptive selection was not found in their analyses. For the same reason, they did not detect evolutionary bistability. In contrast, other studies on fisheries-induced evolution did report the presence of bistability in some traits (Gårdmark and Dieckmann, 2006; de Roos et al., 2006; Boukal et al., 2008). Our study appears to be the first in which evolutionary bistability co-occurs with disruptive selection, and consequently such bistability can be interpreted as an early warning signal for potential disruptive selection (see Fig. 3).

In line with these findings and explanations, our results have also shown that populations with demographic conditions that penalize large individuals and/or favor small individuals are more sensitive to disruptive selection. This is because such populations are naturally prone to early maturation, strengthening the impacts of the tradeoffs in growth and fecundity that turn selection disruptive. Therefore, there are three different ways to promote the mechanism that turns selection disruptive via growth and fecundity tradeoffs: first, the tradeoffs themselves may be strong due to physiological reasons; second, fishing mortality may select for early maturation, making the impacts of those tradeoffs strong; and third, a stock's other demographic and environmental conditions may predispose it to early maturation. Overall, this pattern of chasing the benefits of early maturation while avoiding the costs in growth and fecundity can be considered as an important general mechanism for the origin of dimorphism in exploited fish populations and other coevolving systems (e.g., Zhang et al., 2013).

Our study can be expanded in several directions. First, the target fish stock is the only one component in its embedding ecosystem, where feedbacks to and from its resources and/or predator species intertwine in a complicated web. However, considering these feedbacks could significantly complicate the model and its results. Second, energy-budget approaches can be used for formulating the tradeoffs due to early maturation. Finally, as fishing fleets in many regions of the world are composed of high-technology large commercial boats and low-technology small private boats, the fishery component in this coevolving stock-fishery system could have experienced selective pressures promoting the coexistence and divergence of different fleet segments. In other words, the fleet can experience an analogous disruptive selection and adaptive diversification, as suggested by Dercole et al. (2010) and illustrated by standard eco-evolutionary predator-prey models (Doebeli and Dieckmann, 2000; Landi et al., 2013); this warrants future research and model extensions. Specifically, fishery dynamics could happen at many levels: at the level of the fleet (adaptive harvesting on a short timescale, fleet size and structure on an intermediate timescale, and technological adaptation on a longer timescale; Egas et al., 2005), at the level of fishing strategy (constant effort, fixed quota, or fixed stock size; Hilborn and Walters, 1992), and/or at the level of fishing regulations (limitations on the size and maturity of target individuals; Cole and Ward, 1994; Matsumura et al., 2011). Here we have examined only the simplest setting, that is, adaptive harvesting with a constant-effort strategy. To detect disruptive selection on the fishery, adjustments in fleet size, fleet structure, and fleet technology must be explicitly modeled. As a starting point, the degree of harvest specialization in our model, Eq. (1b), could be interpreted as characterizing the technological level of the fleet (affecting, e.g., the probability of locating aggregations of fish, catchability, and/or the efficiency of handling and transporting the catch). On this basis, this parameter could be used as an adaptive trait of the fishery using the framework of adaptive dynamics theory (Dercole et al., 2008, 2010).

An ultimate target of fishery management is to increase sustainable yield (e.g., Heino, 1998). This raises the question of whether fisheries-induced disruptive selection could, and should, be managed: as such selection pressures result from the interplay between natural selection and fishing mortality (Carlson et al., 2007; Edeline et al., 2007, 2009), they are human-induced and may arguably be controlled by fishing policies and fleet and harvest regulations. In practice, this can be achieved through legal limitations and incentives. Our results show that sustainable yield can slightly increase after diversification when only large individuals are targeted (Fig. 7b), even though it still remains far below the maximum sustainable yield obtained at low fishing mortality when the stock is monomorphic. As many fish stocks are still overexploited, being managed considerably below their maximum sustainable yield, our findings imply that diversification triggered by fisheries-induced disruptive selection under high fishing mortality might slightly increase the yield from its level before diversification, if only large individuals are targeted. However, our results also suggest that such a population dimorphism can be taken as a sign of extreme harvesting pressure, as trait diversification is a way for species to escape from severe selection pressures resulting from human exploitation. Hence, when such a pattern is observed, our analysis suggests that sustainable yield can usually be improved by reducing fishing mortality.

Fisheries-induced disruptive selection could also increase phenotypic variability (Edeline et al., 2009), without promoting lifehistory dimorphism: favoring extreme phenotypes may just widen an existing population polymorphism. This could have positive consequences beyond those analyzed in our study, since higher variability makes a population more reactive to future adaptation needs. This means that the population can react more promptly to any rapid changes in its environmental conditions, both for natural and anthropogenic causes. In other words, fisheries-induced disruptive selection could lead to a better capacity of an exploited stock to cope with environmental disturbances and changes (Roff, 1997).

In summary, fisheries-induced disruptive selection can indicate overexploitation, can slightly increase or decrease the yield depending on the adopted fishing policy, and can enhance a stock's resilience to abrupt changes in its environmental conditions. Weighting these three aspects, decision makers can manage a fishery in pursuit of their economic, social, and conservation objectives.

\section{Acknowledgments}

P.L. is grateful to Fabio Dercole and Sergio Rinaldi for precious comments and discussion. This work has been developed during the Southern African Young Scientists Summer Program (SA-YSSP), for the organization and funding of which we thank the Department of Science and Technology (DST, South Africa), the National Research Foundation (NRF, South Africa), the University of the Free State (UFS, South Africa), and the International Institute for Applied Systems Analysis (IIASA, Austria). P.L. acknowledges funding by the Ministry of Education, Universities and Research, under the project FIRB Futuro in Ricerca "Modeling and Analysis of Innovation and Competition Processes", contract RBFR08TIA4, as well as by the Evolution and Ecology Program (EEP, IIASA) C.H. acknowledges funding by the South African National Research Foundation (contracts 76912, 81825 and 89967). U.D. acknowledges funding by the Sixth Framework Program of the European Commission, the European Science Foundation, the Austrian Science Fund, the Austrian Ministry of Science and Research, and the Vienna Science and Technology Fund. The authors are also grateful to two anonymous reviewers, whose comments and suggestions contributed to improve the quality of this work.

\section{Appendix A}

In this appendix, we specify the population dynamics and the evolutionary dynamics of a dimorphic stock, with population 
densities $\mathbf{N}_{x}=\left(N_{i x}\right)$ for individuals with an early-maturation probability $x$ and of $\mathbf{N}_{y}=\left(N_{i y}\right)$ for individuals with an early-maturation probability $y$. The dimorphic population dynamics are given by

$$
\begin{aligned}
& \dot{N}_{1 x}= f_{\tilde{2}} N_{\tilde{2} x}+f_{\tilde{3}}(x) N_{\tilde{3} x}+f_{3} N_{3 x}-m_{1} N_{1 x}\left(N_{1 x}+N_{1 y}\right) \\
&-r_{1} N_{1 x}-F \alpha_{1} h_{1}\left(\mathbf{N}_{x}, \mathbf{N}_{y}\right) N_{1 x}, \\
& \dot{N}_{2 x}=(1-x) r_{1} N_{1 x}-m_{2} N_{2 x}-r_{2} N_{2 x}-F \alpha_{2} h_{2}\left(\mathbf{N}_{x}, \mathbf{N}_{y}\right) N_{2 x}, \\
& \dot{N}_{\tilde{2} x}= x r_{1} N_{1 x}-m_{\tilde{2}}(x) N_{\tilde{2} x}-r_{\tilde{2}}(x) N_{\tilde{2} x}-F \alpha_{\tilde{2}} h_{\tilde{2}}\left(\mathbf{N}_{x}, \mathbf{N}_{y}\right) N_{\tilde{2} x}, \\
& \dot{N}_{3 x}= r_{2} N_{2 x}-m_{3} N_{3 x}-F \alpha_{3} h_{3}\left(\mathbf{N}_{x}, \mathbf{N}_{y}\right) N_{3 x}, \\
& \dot{N}_{\tilde{3} x}= r_{\tilde{2}}(x) N_{\tilde{2} x}-m_{\tilde{3}} N_{\tilde{3} x}-F \alpha_{\tilde{3}} h_{\tilde{3}}\left(\mathbf{N}_{x}, \mathbf{N}_{y}\right) N_{\tilde{3} x}, \\
& \dot{N}_{1 y}= f_{\tilde{2}} N_{\tilde{2} y}+f_{\tilde{3}}(y) N_{\tilde{3} y}+f_{3} N_{3 y}-m_{1} N_{1 y}\left(N_{1 x}+N_{1 y}\right) \\
&-r_{1} N_{1 y}-F \alpha_{1} h_{1}\left(\mathbf{N}_{x}, \mathbf{N}_{y}\right) N_{1 y}, \\
& \dot{N}_{2 y}=(1-y) r_{1} N_{1 y}-m_{2} N_{2 y}-r_{2} N_{2 y}-F \alpha_{2} h_{2}\left(\mathbf{N}_{x}, \mathbf{N}_{y}\right) N_{2 y}, \\
& \dot{N}_{\tilde{2} y}= y r_{1} N_{1 y}-m_{\tilde{2}}(y) N_{\tilde{2} y}-r_{\tilde{2}}(y) N_{\tilde{2} y}-F \alpha_{\tilde{2}} h_{\tilde{2}}\left(\mathbf{N}_{x}, \mathbf{N}_{y}\right) N_{\tilde{2} y}, \\
& \dot{N}_{3 y}= r_{2} N_{2 y}-m_{3} N_{3 y}-F \alpha_{3} h_{3}\left(\mathbf{N}_{x}, \mathbf{N}_{y}\right) N_{3 y}, \\
& \dot{N}_{\tilde{3} y}= r_{\tilde{2}}(y) N_{\tilde{2} y}-m_{\tilde{3}} N_{\tilde{3} y}-F \alpha_{\tilde{3}} h_{\tilde{3}}\left(\mathbf{N}_{x}, \mathbf{N}_{y}\right) N_{\tilde{3} y}, \\
& \text { where }
\end{aligned}
$$

$$
h_{i}\left(\mathbf{N}_{x}, \mathbf{N}_{y}\right)=\frac{\left(\alpha_{i} w_{i}\left(N_{i x}+N_{i y}\right)\right)^{\gamma}}{\sum_{j}\left(\alpha_{j} w_{j}\left(N_{j x}+N_{j y}\right)\right)^{\gamma}},
$$

with the sum extending over all five stock components $j=1,2, \tilde{2}, 3$, or $\tilde{3}$.

Indicating by $x^{\prime}$ and $y^{\prime}$ the trait values of mutants appearing in a population with resident trait values $x$ and $y$ we obtain the basic reproduction ratios of such mutants as

$R_{0}\left(x, y, x^{\prime}\right)=r_{1} D_{1}\left\{\left(1-x^{\prime}\right) r_{2} D_{2} D_{3} f_{3}+x^{\prime}\left[D_{\tilde{2} x} f_{\tilde{2}}+r_{\tilde{2}}\left(x^{\prime}\right) D_{\tilde{2} x} D_{\tilde{3}} f_{\tilde{3}}\left(x^{\prime}\right)\right]\right\}$, $R_{0}\left(x, y, y^{\prime}\right)=r_{1} D_{1}\left\{\left(1-y^{\prime}\right) r_{2} D_{2} D_{3} f_{3}+y^{\prime}\left[D_{\tilde{2} y} f_{\tilde{2}}+r_{\tilde{2}}\left(y^{\prime}\right) D_{\tilde{2} y} D_{\tilde{3}} f_{\tilde{3}}\left(y^{\prime}\right)\right]\right\}$,

where $D_{1}=\left[m_{1}\left(N_{1 x}^{*}+N_{1 y}^{*}\right)+r_{1}+F \alpha_{1} h_{1}\left(\mathbf{N}_{x}^{*}, \mathbf{N}_{y}^{*}\right)\right]^{-1}, D_{2}=\left[m_{2}+r_{2}+\right.$ $\left.F \alpha_{2} h_{2}\left(\mathbf{N}_{x}^{*}, \mathbf{N}_{y}^{*}\right)\right]^{-1}, \quad D_{\tilde{2} x}=\left[m_{\tilde{2}}\left(x^{\prime}\right)+r_{\tilde{2}}\left(x^{\prime}\right)+F \alpha_{\tilde{2}} h_{\tilde{2}}\left(\mathbf{N}_{x}^{*}, \mathbf{N}_{y}^{*}\right)\right]^{-1}, \quad D_{\tilde{2} y}=$ $\left[m_{\tilde{2}}\left(y^{\prime}\right)+r_{\tilde{2}}\left(y^{\prime}\right)+F \alpha_{\tilde{2}} h_{\tilde{2}}\left(\mathbf{N}_{x}^{*}, \mathbf{N}_{y}^{*}\right)\right]^{-1}, \quad D_{3}=\left[m_{3}+F \alpha_{3} h_{3}\left(\mathbf{N}_{x}^{*}, \mathbf{N}_{y}^{*}\right)\right]^{-1}$, and $D_{\tilde{3}}=\left[m_{\tilde{3}}+F \alpha_{\tilde{3}} h_{\tilde{3}}\left(\mathbf{N}_{x}^{*}, \mathbf{N}_{y}^{*}\right)\right]^{-1}$ are the average durations spent by individuals in the stock components, and $\left(\mathbf{N}_{x}^{*}, \mathbf{N}_{y}^{*}\right)$ are the population densities at the dimorphic demographic equilibrium.

On the evolutionary timescale, the traits $x$ and $y$ evolve following a two-dimensional canonical equation

$$
\dot{x}=\left.\epsilon k_{x} \sum_{i} N_{i x}^{*} \frac{\partial R_{0}\left(x, y, x^{\prime}\right)}{\partial x^{\prime}}\right|_{x^{\prime}=x}, \quad \dot{y}=\left.\epsilon k_{y} \sum_{i} N_{i y}^{*} \frac{\partial R_{0}\left(x, y, y^{\prime}\right)}{\partial y^{\prime}}\right|_{y^{\prime}=y},
$$

where $\epsilon$ is the time-scaling parameter, separating the (slow) evolutionary dynamics from the (fast) demographic dynamics, $k_{x}$ and $k_{y}$ are half the product of probability and variance of mutations, scaling the speed of evolutionary dynamics in $x$ and $y$, respectively, and the sum extends over all five stock components $i=1,2, \tilde{2}, 3$, and $\tilde{3}$. These dimorphic dynamics converges to the dimorphic evolutionary equilibrium $\left(x_{\mathrm{D}}^{*}, y_{\mathrm{D}}^{*}\right)$.

Finally, the sustainable yield of the dimorphic stock with trait values $\left(x_{\mathrm{D}}^{*}, y_{\mathrm{D}}^{*}\right)$ is given by

$Y_{\mathrm{D}}=\left.\sum_{i} F \alpha_{i} h_{i}\left(\mathbf{N}_{x}^{*}, \mathbf{N}_{y}^{*}\right)\left(N_{i x}^{*}+N_{i y}^{*}\right) w_{i}\right|_{x=x_{\mathrm{D}}^{*}, y=y_{\mathrm{D}}^{*}}$,

with the sum extending over all five stock components $i=1,2, \tilde{2}, 3$, and $\tilde{3}$.

\section{References}

Abrams, P.A., Harada, Y., Matsuda, H., 1993. On the relationship between quantitative genetic and ESS models. Evolution 47, 982-985.

Abrams, P.A., Rowe, L., 1996. The effects of predation on the age and size of maturity of prey. Evolution 50, 1052-1061.
Ajiad, A., Jakobsen, T., Nakken, O., 1999. Sexual difference in maturation of Northeast Arctic cod. J. Northwest Atlant. Fish. Sci. 25, 1-15.

Allgower, E.L., Georg, K., 2003. Introduction to Numerical Continuation Methods. SIAM Class. Appl. Math., http://dx.doi.org/10.1137/1.9780898719154.

Ashley, M.V., Willson, M.F., Pergams, O.R.W., O'Dowd, D.J., Gende, S.M., Brown, J.S., 2003. Evolutionarily enlightened management. Biol. Conserv. 111, 115-123.

Barot, S., Heino, M., O'Brien, L., Dieckmann, U., 2004. Long-term trend in the maturation reaction norm of two cod stocks. Ecol. Appl. 14, 1257-1271.

Bodin, M., Brännström, Å., Dieckmann, U., 2012. A systematic overview of harvesting-induced maturation evolution in predator-prey systems with three different life-history tradeoffs. Bull. Math. Biol. 74, 2842-2860.

Boukal, D.S., Dunlop, E.S., Heino, M., Dieckmann, U., 2008. Fisheries-induced evolution of body size and other life history traits: the impact of gear selectivity, in: ICES CM/F:07.

Carlson, S.M., Edeline, E., Vøllestad, L.A., Haugen, T.O., Winfield, I.J., Fletcher, J.M., James, J.B., Stenseth, N.C., 2007. Four decades of opposing natural and humaninduced artificial selection acting on Windermere pike (Esox lucius). Ecol. Lett. $10,512-521$

Champagnat, N., Ferrière, R., Méléard, S., 2006. Unifying evolutionary dynamics: from individual stochastic processes to macroscopic models. Theor. Popul. Biol. 69, 297-321.

Cole, R.A., Ward, F.A., 1994. Optimum fisheries management policy: angler opportunity versus angler benefit. North Am. J. Fish. Manag. 14, 22-33.

Darimont, C.T., Carlson, S.M., Kinnison, M.T., Paquet, P.C., Reimchen, T.E., Wilmers, C.C., 2009. Human predators outpace other agents of trait change in the wild. Proc. Natl. Acad. Sci. USA 106, 952-954.

de Roos, A.M., Boukal, D.S., Person, L., 2006. Evolutionary regime shifts in age and size at maturation of exploited fish stoks. Proc. R. Soc. Lond. Ser. B 273, 1873-1880.

Dercole, F., Dieckmann, U., Obersteiner, M., Rinaldi, S., 2008. Adaptive dynamics and technological change. Technovation 28, 335-348.

Dercole, F., Ferrière, R., Rinaldi, S., 2002. Ecological bistability and evolutionary reversals under asymmetrical competition. Evolution 56, 1081-1090.

Dercole, F., Prieu, C., Rinaldi, S., 2010. Technological change and fisheries sustainability: the point of view of adaptive dynamics. Ecol. Model. 221, 379-387.

Dercole, F., Rinaldi, S., 2008. Analysis of Evolutionary Processes: The Adaptive Dynamics Approach and Its Applications. Princeton University Press, Princeton, NJ.

Dieckmann, U., Heino, M., 2007. Probabilistic maturation reaction norms: their history, strengths, and limitations. Mar. Ecol. Prog. Ser. 335, 253-269.

Dieckmann, U., Heino, M., Parvinen, K., 2006. The adaptive dynamics of functionvalued traits. J. Theor. Biol. 241, 370-389.

Dieckmann, U., Heino, M., Rijnsdorp, A.D., 2009. The dawn of Darwinian fishery management. ICES Insight 46, 34-43.

Dieckmann, U., Law, R., 1996. The dynamical theory of coevolution: a derivation from stochastic ecological processes. J. Math. Biol. 34, 579-612.

Doebeli, M., Dieckmann, U., 2000. Evolutionary branching and sympatric speciation caused by different types of ecological interactions. Am. Nat. 156, 77-101.

Dunlop, E.S., Heino, M., Dieckmann, U., 2009. Eco-genetic modeling of contemporary life-history evolution. Ecol. Appl. 19, 1815-1834.

Edeline, E. Carlson, S.M., Stige, L.C., Winfield, I.J., Fletcher, J.M., James, J.B., Haugen, T.O., Vøllestad, L.A., Stenseth, N.C., 2007. Trait changes in a harvested population are driven by a dynamic tug-of-war between natural and harvest selection. Proc. Natl. Acad. Sci. USA 104, 15799-15804.

Edeline, E., Le Rouzic, A., Winfield, I.J., Fletcher, J.M., Ben James, J., Stenseth, N.C., Vøllestad, L.A., 2009. Harvest-induced disruptive selection increases variance in fitness-related traits. Proc. R. Soc. Lond. Ser. B 276, 4163-4171.

Egas, M., Sabelis, M.W., Dieckmann, U., 2005. Evolution of specialization and ecological character displacement of herbivores along a gradient of plant quality. Evolution 3, 507-520.

Engelhard, G.H., Heino, M., 2004. Maturity changes in Norwegian spring-spawning herring Clupea harengus: compensatory or evolutionary responses. Mar. Ecol. Prog. Ser. 272, 245-256.

Ernande, B., Dieckmann, U., Heino, M., 2004. Adaptive changes in harvested populations: plasticity and evolution of age and size at maturation. Proc. R. Soc. Lond. Ser. B 271, 415-423.

Eshel, I., 1983. Evolutionary and continuous stability. J. Theor. Biol. 103, 99-111.

Fenberg, P.B., Roy, K., 2008. Ecological and evolutionary consequences of sizeselective harvesting: how much do we know?. Mol. Ecol. 17, 209-220.

Fromentin, J.M., Powers, J.E., 2005. Atlantic bluefin tuna: population dynamics, ecology, fisheries, and management. Fish Fish. 6, 281-306.

Geritz, S.A.H., Kisdi, E., Meszéna, G., Metz, J.A.J., 1998. Evolutionarily singular strategies and the adaptive growth and branching of the evolutionary tree. Evolution. Ecol. 12, 35-57.

Geritz, S.A.H., Metz, J.A.J., Kisdi, E., Meszéna, G., 1997. The dynamics of adaptation and evolutionary branching. Phys. Rev. Lett. 78, 2024-2027.

Gårdmark, A., Dieckmann, U., 2006. Disparate maturation adaptations to sizedependent mortality. Proc. R. Soc. Lond. Ser. B 273, 2185-2192.

Gross, M.R., 1985. Disruptive selection for alternative life histories in salmon. Nature 313, 47-48.

Gross, M.R., 1996. Alternative reproductive strategies and tactics: diversity within sexes. Trends Ecol. Evol. 11, 92-98.

Hannesson, R., 2002. The economics of fisheries. In: Hart, P.J.B., Reynolds, J. (Eds.), Handbook of Fish Biology and Fisheries. Blackwell Science, Oxford, UK.

Hart, P.J.B., Reynolds, J. (Eds.), 2002. Handbook of Fish Biology and Fisheries. Blackwell Science, Oxford, UK. 
Heino, M., 1998. Management of evolving fish stocks. Can. J. Fish. Aquat. Sci. 55, 1971-1982.

Heino, M., Godø, O.R., 2002. Fisheries-induced selection pressure in the context of sustainable fisheries. Bull. Mar. Sci. 70, 639-656.

Hilborn, R., Walters, C.J., 1992. Quantitative Fisheries Stock Assessment: Choice, Dynamics and Uncertainty. Chapman and Hall, New York.

Hutchings, J.A., Fraser, D.J., 2008. The nature of fisheries- and farming-induced evolution. Mol. Ecol. 17, 294-313.

Hutchings, J.A., Reynolds, J.D., 2004. Marine fish population collapses: consequences for recovery and extinction risk. Bioscience 54, 297-309.

Jennings, S., Kaiser, M.J., 1998. The effects of fishing on marine ecosystems. Adv. Mar. Biol. 34, 201-352.

Jørgensen, C., Enberg, K., Dunlop, E.S., Arlinghaus, R., Boukal, D., Brander, K., Ernande, B., Gårdmark, A., Johnson, F., Matsumura, S., Pardoe, H., Raab, K., Silva, A., Vainikka, A., Dieckmann, U., Heino, M., Rijnsdorp, A.D., 2007. Managing evolving fish stocks. Science 318, 1247-1248.

Keller, I., Wagner, C.E., Greuter, L., Mwaiko, S., Selz, O.M., Sivasundar, A., Wittwer, S., Seehausen, O., 2013. Population genomic signatures of divergent adaptation, gene flow and hybrid speciation in the rapid radiation of Lake Victoria cichlid fishes. Mol. Ecol. 22, 2848-2863.

Kuznetsov, Yu.A., 2004. Elements of Applied Bifurcation Theory, 3rd edition. Springer Verlag, New York.

Landi, P., Dercole, F., Rinaldi, S., 2013. Branching scenarios in eco-evolutionary preypredator models. SIAM J. Appl. Math. 73, 1634-1658.

Law, R., 1979. Optimal life-histories under age-specific predation. Am. Nat. 114, $399-417$.

Law, R., 2000. Fishing, selection and phenotypic evolution. ICES J. Mar. Sci. 57, 659-669.

Law, R., Grey, D.R., 1989. Evolution of yields from populations with age-specific cropping. Evolution. Ecol. 3, 343-359.

Mansueti, R.J., 1961. Age, growth, and movements of the striped bass, Roccus saxatilis taken in size selective fishing gear in maryland. Chesapeake Sci. 2, 9-36.

Matsumura, S., Arlinghaus, R., Dieckmann, U., 2011. Assessing evolutionary consequences of size-selective recreational fishing on multiple life-history traits, with an application to northern pike (Esox lucius). Evolution. Ecol. 25, 711-735.
Maynard Smith, J., 1966. Sympatric speciation. Am. Nat. 100, 637-650.

McAllister, M.K., Peterman, R.M., 1992. Decision analysis of a large-scale fishing experiment designed to test for a genetic effect of size-selective fishing on British Columbia pink salmon (Oncorhynchus gorbuscha). Can. J. Fish. Aquat. Sci. 49, 1305-1314.

Opdal, A.F., 2010. Fisheries change spawning ground distribution of northeast Arctic cod. Biol. Lett. 6, 261-264.

Palumbi, S.R., 2001. Humans as the world's greatest evolutionary force. Science 293, 1786-1790.

Poos, J.J., Brännström, Å., Dieckmann, U., 2011. Harvest-induced maturation evolution under different life-history trade-offs and harvesting regimes. J. Theor. Biol. 279, 102-112.

Roff, D.A., 1983. An allocation model of growth and reproduction in fish. Can. J. Fish. Aquat. Sci. 40, 1395-1404.

Roff, D.A., 1997. Evolutionary Quantitative Genetics. Chapman and Hall, New York. Salthaug, A., 2001. Adjustment of commercial trawling effort for Atlantic cod, Gadus morhua, due to increasing catching efficiency. Fish. Bull. 99, 338-342.

Sinclair, A., 1992. Fish distribution and partial recruitment: the case of eastern Scotian Shelf cod. J. Northwest Atlant. Fish. Sci. 13, 15-24.

Stearns, S.C., 1992. The Evolution of Life Histories. Oxford University Press, New York.

Stokes, T.K., McGlade, J.M., Law, R., 1993. The Exploitation of Evolving Resources. Springer-Verlag, Berlin, Germany.

Swain, D.P., Wade, E.J., 1993. Density-dependent geographic distribution of Atlantic cod (Gadus morhua) in the southern Gulf of St. Lawrence. Can. J. Fish. Aquat. Sci. 50, 725-733.

Walters, C.J., Martell, S.J.D., 2004. Fisheries Ecology and Management. Princeton University Press, Princeton, NJ.

Zhang, F., Hui, C., Pauw, A., 2013. Adaptive divergence in Darwin's race: how coevolution can generate trait diversity in a pollination system. Evolution 67, $548-560$.

Zhou, S., Smith, A.D.M., Punt, A.E., Richardson, A.J., Gibbs, M., Fulton, E.A., Pascoe, S., Bulman, C., Bayliss, P., Sainsbury, K., 2010. Ecosystem-based fisheries management requires a change to the selective fishing philosophy. Proc. Natl. Acad. Sci. USA 107, 9485-9489. 